\title{
Evaluation of wall friction models for riser flow
}

Article in Powder Technology · December 2017

Impact Factor: 2.35

READ

1

4 authors, including:

Jan Hendrik Cloete

Norwegian University of Science and Techn...

7 PUBLICATIONS 1 CITATION

SEE PROFILE 


\title{
Evaluation of wall friction models for riser flow
}

\author{
Jan Hendrik Cloete ${ }^{1}$, Schalk Cloete ${ }^{2}$, Stefan Radl ${ }^{3}$, Shahriar Amini ${ }^{1,2^{*}}$
}

1) Department of Energy and Process Engineering, Norwegian University of Science and Technology (NTNU), NO-7491 Trondheim, Norway

2) Flow Technology Department, SINTEF Materials and Chemistry, NO-7465 Trondheim, Norway

3) Institute of Process and Particle Engineering, Graz University of Technology, Inffeldgasse 13/III, Graz, Austria

*Corresponding author. Email: shahriar.amini@sintef.no

Address: SINTEF Materials and Chemistry, Richard Birkelands Vei 3, 7034 Trondheim, Norway, Phone: +4746639721

\begin{abstract}
Two different approaches for modelling the particle-wall collisions, the frequently employed Johnson \& Jackson model and the recently proposed Schneiderbauer model, were evaluated in a fluidized bed riser by comparing simulation results to experimental data over a range of fluidization velocities and solids fluxes. For the Johnson \& Jackson model, it was shown that partial slip settings recommended for denser fluidization conditions (a specularity coefficient in the order of 0.1) failed to predict cluster formation at the walls at higher gas flow rates due to unrealistically large granular temperature generation in the near-wall regions. By reducing wall friction to settings approaching a free-slip condition (specularity coefficient in the order of 0.001), this problem is overcome by eliminating excessive granular temperature generation from over-predicted strain rates at the walls. However, this approach results in an overestimation of the downward velocity of the clusters at the wall in dense cases. Despite this shortcoming, predictions are remarkably accurate for most of the cases. The Schneiderbauer model, with model parameters close to recommended settings, performs similarly well for most of the cases, slightly under-predicting cluster formation at the walls in the dilute cases. Generally, it also predicts more realistic flow behaviour since it prevents dense clusters from falling rapidly at the walls. The Schneiderbauer wall friction model is therefore recommended for use in future studies of risers, since it is able to deliver reasonable results over a wider range of flow conditions than the Johnson and Jackson model, using a single set of friction parameters. Furthermore, it has the benefit of using experimentally measurable quantities as input.
\end{abstract}

Keywords: Fluidised bed, Riser, Kinetic Theory of Granular Flow, Wall friction model, Particle-wall collisions, Two-Fluid Model 


\section{Introduction}

Circulating fluidised beds (CFBs) are used in the chemical, petrochemical, energy and metallurgical industries for applications such as fluid catalytic cracking (FCC), coal and biomass gasification and chemical looping combustion (CLC). In the riser section of the CFB the solid particles are transported vertically in a gas stream, enabling high gas throughput rates and excellent contact between the gas and solid phases for reactions and heat transfer. It is known that during this transport process the solids will gather in clusters of particles due to local instabilities and that this behaviour has an important impact on the hydrodynamic and reactive performance of these reactors [1]. Due to this complex multiphase flow behaviour, these processes face many challenges regarding operation, design and scale-up.

Computational fluid dynamics (CFD) has emerged as a valuable tool through which the understanding of these systems can be improved. A common approach is to use a two-fluid model (TFM) where the particle phase is assumed to be continuous [2-4]. The solid phase is governed by the kinetic theory of granular flow (KTGF), where the behaviour of the solid phase is analogous to the kinetic theory for gases and the granular temperature represents the kinetic energy of unresolved random motions of the solid particles. It has been shown that the TFM can qualitatively capture the phenomena observed in experimental setups of fluidised beds, including the formation of particle phase clusters. Good quantitative comparisons have also been made with experimental data for certain conditions, but in many cases it has proved difficult to achieve a good match between experimental and numerical results in risers $[5,6]$.

Two complicating factors can be identified in risers. Firstly, small particle sizes and large gas velocities are essential to facilitate the transport of solids, demanding small grid sizes and time steps. As a result, risers are computationally demanding to simulate and simplifying assumptions are often used in the literature, such as using 2D simulations and coarse grids. Recent research has placed much emphasis on developing sub-grid models to achieve better results from coarse grid simulations [7-9].

Secondly, particle-wall collisions play a critical role in the behaviour of risers due to the large ratio of wall area to reactor volume and the high velocity of particles typical of risers. There is general agreement in the literature that the choice of wall boundary condition for the solid phase have a significant influence on the overall hydrodynamics of the riser [10, 11]. However, it remains unclear what the correct approach is for including the wall effects, as boundary conditions in the literature include anything from free-slip to no-slip.

The most popular model for the particle-wall boundary condition, by a considerable margin, is that of Johnson and Jackson [12]. At the time of writing, the original paper describing the model had over 700 citations in the literature. This is despite the well-known limitations of the approach $[13,14]$. In the Johnson and Jackson model the effect of particle-wall friction and wall roughness on the shear force is incorporated into a single heuristic constant, called the specularity coefficient. The specularity coefficient is not a physically measurable property and its desired value may change with flow conditions. This is a problem in systems such as 
circulating fluidised beds, which may contain dense and dilute regions, requiring very different specularity coefficients for accurate results in these different regions. The dependence on flow conditions leads to a disparity in the values of the specularity coefficient used in literature studies of risers, with one group assuming partial slip (specularity coefficients of 0.1 to 0.5$)[15,16]$ and another group assuming near free-slip (values smaller or equal to 0.001$)[11,17]$.

Furthermore, the Johnson and Jackson model assumes a linear relationship between the shear stress and the slip velocity. However, it is well known that at high slip velocities all the particles will slide at contact and that the shear stress will be limited by Coulomb friction [1821]. The Johnson and Jackson model therefore tends to over-predict the shear stress and granular temperature generation for rapid flows, which explains the use of very low specularity coefficient values in the literature.

Despite the dominance of the Johnson and Jackson model, there are alternative methods available in literature. Jenkins [18] proposed expressions for the shear stress and granular temperature flux in terms of measurable quantities, the friction coefficient $\left(\mu_{w}\right)$ and the tangential $\left(\beta_{0}\right)$ and normal $\left(e_{w}\right)$ particle-wall restitution coefficients. However, their theory was restricted to the limits of either non-sliding or all-sliding collisions; therefore a-priori knowledge of the flow domain is required for using their model. Jenkins and Louge [22] improved these correlations for the flux of the granular temperature based on computer simulations of Louge [19] for the limits of non-sliding and all-sliding collisions.

Sliding and non-sliding collisions were first linked into one expression by Li and Benyahia [20], who provided an expression for the specularity coefficient based on the friction coefficient, particles-wall restitution coefficients, slip velocity and granular temperature. This approach therefore solves the problem of the specularity coefficient not being a measurable quantity, as well as its dependency on the flow conditions. However, it was recently noted that this approach does not differentiate between sliding and non-sliding collisions in the dissipation term of the boundary condition for the granular temperature, leading to an overprediction of the granular flux in rapid granular flows [13].

The model by Schneiderbauer [21] also included sliding and non-sliding collisions in one expression, dependent on the friction and particle-wall restitution coefficients. However, an improved treatment of the granular flux leads to better comparisons with the simulation data of Louge [19], compared to the work of [20]. Additionally, the model can also account for a boundary moving in a normal direction relative to the flow, making it the only approach suitable to systems with moving parts.

Most recently, Zhao [14] achieved an even better comparison with the data of Louge [19] by also considering the rotational granular temperature of the particles. However, the approach of using a rotational granular temperature is not common practice due to the added 
computational expense of solving an extra conservation equation and the complexity added by the additional closures.

For this reason it can be argued that currently the model by Schneiderbauer [21] is the best alternative for replacing the Johnson and Jackson model [12] as the most commonly used wall-friction model for granular flows. It has the primary advantages of requiring only measurable quantities as input and achieving a very good match with simulation data by Louge [19] by accounting for the effect of a transition from non-sliding to sliding collisions on both the shear stress and the granular flux. Additionally, it retains most of the simplicity that makes the Johnson and Jackson model popular.

The potential benefit of the Schneiderbauer wall-friction model has been demonstrated in a spouted bed [23] for fluidised beds. However, its true advantage is expected to be best illustrated in risers, where rapid granular flow occurs at the walls. For this reason, this paper will aim to evaluate the Schneiderbauer model as an alternative to the Johnson and Jackson model by comparing numerical results with experimental data in risers over a range of superficial gas velocities and solids fluxes.

\section{Simulations}

The setup for the numerical simulations is similar to that used in a previous study [10], but the most important equations are repeated here for clarity. A more detailed discussion of the equations used can be found in [24].

\subsection{Model equations}

This study will use Eulerian two-fluid modelling to simulate the flow behaviour inside a riser. In this modelling framework, the gas and solid phases are each treated as a separate, continuous phase, with conservation equations being solved for each phase. The continuity equations for the gas and solids are written as follows:

$\frac{\partial}{\partial t}\left(\alpha_{g} \rho_{g}\right)+\nabla \cdot\left(\alpha_{g} \rho_{g} \vec{v}_{g}\right)=0$

Equation 1

$\frac{\partial}{\partial t}\left(\alpha_{s} \rho_{s}\right)+\nabla \cdot\left(\alpha_{s} \rho_{s} \vec{v}_{s}\right)=0$

Equation 2

The momentum conservation equation for the gas phase is

$\frac{\partial}{\partial t}\left(\alpha_{g} \rho_{g} \vec{v}_{g}\right)+\nabla \cdot\left(\alpha_{g} \rho_{g} \vec{v}_{g} \vec{v}_{g}\right)=-\alpha_{g} \nabla p+\nabla \cdot \overline{\bar{\tau}}_{g}+\alpha_{g} \rho_{g} \vec{g}+K_{s q}\left(\vec{v}_{s}-\vec{v}_{g}\right)$

Equation 3

And for the solids

$\frac{\partial}{\partial t}\left(\alpha_{s} \rho_{s} \vec{v}_{s}\right)+\nabla \cdot\left(\alpha_{s} \rho_{s} \vec{v}_{s} \vec{v}_{s}\right)=-\alpha_{s} \nabla p-\nabla p_{s}+\nabla \cdot \overline{\bar{\tau}}_{s}+\alpha_{s} \rho_{s} \vec{g}+K_{g s}\left(\vec{v}_{g}-\vec{v}_{s}\right)$

Equation 4

The inter-phase momentum exchange coefficient $\left(K_{g s}=K_{s g}\right)$ was modelled using the Huilin-Gidaspow drag model [25]. It combines the Wen-Yu model [26] with the Ergun equation at high solids volume fractions and uses a blending function to smooth out the discontinuity between the two equations. 
The solids stresses in the particle phase momentum equation are solved based on the Kinetic Theory of Granular Flow. In this approach the random motion of the particles is likened to the thermal motion of the molecules in a gas. The kinetic energy of these random fluctuations is quantified by the granular temperature, for which an additional conservation equation is solved.

$$
\frac{3}{2}\left[\frac{\partial}{\partial t}\left(\alpha_{s} \rho_{s} \Theta_{s}\right)+\nabla \cdot\left(\alpha_{s} \rho_{s} \vec{v}_{s} \Theta_{s}\right)\right]=\left(-p_{s} \overline{\bar{I}}+\overline{\bar{\tau}}_{s}\right): \nabla \vec{v}_{s}+\nabla \cdot\left(k_{\Theta_{s}} \nabla \Theta_{s}\right)-\gamma_{\Theta_{s}}+\phi_{g s}
$$

The first term on the right hand side contains the normal and shear solids stresses, with closures required for the solids pressure [4] and the shear [2] and bulk [4] viscosities. The other terms on the right hand side are the granular conductivity [2] $\left(k_{\Theta_{s}} \nabla \Theta_{s}\right)$, the collisional dissipation of energy [4] $\left(\gamma_{\Theta_{s}}\right)$ and the momentum exchange between the random particle motions and the gas phase [2] $\left(\phi_{g s}\right)$.

Of particular significance for this paper are the equations used for modelling the particle-wall interaction. The contribution of the wall comes in a shear stress term that is included in the particle phase momentum equation (Equation 4) and a granular flux term that is included in the granular temperature equation (Equation 5).

The first formulation used is that by Johnson and Jackson [12], for which the expressions for the shear stress and the granular flux are:

$$
\begin{aligned}
& \vec{\tau}_{s}=-\frac{\pi}{6} \sqrt{3} \phi \frac{\alpha_{s}}{\alpha_{s, \max }} \rho_{s} g_{0} \sqrt{\Theta_{s}} \vec{v}_{s, \mid} \\
& q_{s}=\vec{\tau}_{s} \cdot \vec{v}_{s, \mid}-\frac{\pi}{4} \sqrt{3} \frac{\alpha_{s}}{\alpha_{s, \max }}\left(1-e_{w}{ }^{2}\right) \rho_{s} g_{0} \Theta_{s}{ }^{\frac{3}{2}}
\end{aligned}
$$

where the input parameters are the specularity coefficient $(\phi)$ and the particle-wall restitution coefficient $\left(e_{w}\right)$.

For the Schneiderbauer model [21], the following set of equations are used:

$$
\begin{aligned}
& \vec{\tau}_{s}=-n_{w} \mu_{w} \alpha_{s} \rho_{s} g_{0} \Theta_{s} \operatorname{erf}\left(\overline{v_{s}}\right) \frac{\vec{v}_{s, \mid}}{\left\|\vec{v}_{s, \mid}\right\|} \\
& q_{s}=\vec{\tau}_{s} \cdot \vec{v}_{s, \mid}-\frac{\alpha_{s} \rho_{s} g_{0} n_{w} \sqrt{\Theta_{s}}}{\sqrt{2} \mu_{0}^{2} \sqrt{\pi}} \exp \left(-{\overline{v_{s}}}^{2}\right) \times \\
& \left(\mu_{w}\left(2 \mu_{w}\left\|\vec{v}_{s, \|}\right\|^{2}\left(2 n_{w}-\mu_{0}\right)+\Theta_{s}\left(14 \mu_{w} n_{w}-4 \mu_{0}\left(1+\mu_{w}\right)-6 \mu_{w} \mu_{0}^{2} n_{w}\right)\right)\right. \\
& \left.+\mu_{0}^{2} \sqrt{\Theta_{s}} \exp \left(\bar{v}_{s}^{2}\right)\left(\sqrt{\Theta_{s}}\left(4\left(n_{w}-1\right)+6 \mu_{w}^{2} n_{w}\right)-\sqrt{2 \pi} \mu_{w}\left\|\vec{v}_{s, \|}\right\| \operatorname{erf}\left(\overline{v_{s}}\right)\right)\right) \\
& n_{w}=\frac{1}{2}\left(1+e_{w}\right)
\end{aligned}
$$


$\overline{v_{s}}=\frac{\left\|\vec{v}_{s, l}\right\|}{\sqrt{2 \Theta_{s}} \mu_{0}}$

Equation 11

$\mu_{0}=\frac{7}{2} \frac{1+e_{w}}{1+\beta_{0}} \mu_{w}$

Equation 12

where the input values are the friction coefficient $\left(\mu_{w}\right)$, the tangential restitution coefficient $\left(\beta_{0}\right)$ and the normal restitution coefficient $\left(e_{w}\right)$.

The radial distribution function is calculated using the equation by Arastoopour [27] to be consistent with the work by Schneiderbauer [23], where they optimised the three parameters in their wall friction model for a spouted bed.

\subsection{Solver settings}

All simulations are performed using the commercial CFD software, Fluent 16.2.0. Pressurevelocity coupling is performed using the phase-coupled SIMPLE algorithm [28], all other equations are discretised using the QUICK scheme [29]. Second-order implicit time stepping was used since it has been shown previously that time step independent results could not be reached in a similar riser setup using first-order implicit time stepping [24].

\subsection{Simulation geometry}

The simulations are performed on a $800 \mathrm{~mm}$ long section of a riser with a diameter of $76 \mathrm{~mm}$ according to the experiments by Yan and Zhu [30, 31]. A periodic section of the riser is simulated, assuming fully developed flow in the experimental setup.

Due to the large number of simulations that will be required to study the effect of changing the parameters in the Schneiderbauer model at different flow conditions, it was deemed impractical to perform 3D simulations. However, in 2D simulations the ratio of the wall area to reactor volume is significantly reduced compared to the 3D case. It is important to correctly maintain this ratio since the primary purpose of this study is to investigate the effect of the walls. For this reason a recently published 2.5D approach is utilised [32]. In this method a single layer of 3D cells is used, with the cell volume increasing from the riser centre towards the walls to accurately reproduce the geometrical proportions in the $3 \mathrm{D}$ riser, as is done in an axisymmetric simulation. However, in the $2.5 \mathrm{D}$ model the two wedges that subsequently form are linked in the centre by a rectangle to allow particles to be transported across the centre. This solves the primary problem of using axisymmetric simulations for predicting riser flow [32].

This setup allows a much better representation of the riser geometry, while still maintaining the same number of cells as in 2D. An additional benefit of the 2.5D approach is that it does not require the boundary conditions or initial conditions to be adapted, as was done in a previous study [24], to allow for comparison between 2D simulations and experimental data. This is because the $2.5 \mathrm{D}$ model accurately reproduces the radial proportions of the riser 
geometry, except for a slight overestimation of the volume caused by the rectangle connecting the wedges.

Despite the better comparison with experimental data afforded by the 2.5D approach, it was noted by [32] that 2.5D simulations tend to over-predict the solids velocity in the centre of the reactor compared to 3D simulations. This behaviour was also observed in the present study when comparing simulation results to experimental data (Figure 8). However, keeping this behaviour in mind, 2.5D simulations results are still sufficient for the purpose of this study when limiting the comparison with experimental results to the solids velocity profile closer to the wall. In this region, the results are most sensitive to the wall boundary condition and comparison between 2.5D and 3D simulation results are reasonably good. Comparison of 2.5D simulation results and experimental data near the wall is therefore sufficient for the evaluation of different wall boundary condition settings.

A structured grid of $0.67 \times 1.34 \mathrm{~mm}$ cells is used. This grid size was shown earlier to give mesh independent results in combination with second order time discretisation for the riser considered in this study [24].

\subsection{Initial and boundary conditions}

The top and bottom boundary conditions are specified to be translationally periodic in the length of the riser. To be able to maintain the conditions used in the experiments by Yan and Zhu [30, 31], the pressure drop is adjusted to maintain the target gas superficial velocity, as discussed in [24]. Each simulation is initialised with the domain-averaged particle volume fraction for that specific case. The superficial gas velocity and the particle volume fraction used in the simulations correspond to the five cases investigated by Yan and Zhu [30, 31]. These operating conditions are summarised in Table 1.

\begin{tabular}{|l|l|l|l|}
\hline Case & $\begin{array}{c}\text { Superficial } \\
\text { velocity } \\
(\mathrm{m} / \mathrm{s})\end{array}$ & $\begin{array}{c}\text { Solids } \\
\text { flux } \\
\left(\mathrm{kg} / \mathrm{m}^{2} \mathrm{~s}\right)\end{array}$ & $\begin{array}{c}\text { Average } \\
\text { volume } \\
\text { fraction }\end{array}$ \\
\hline 1 & 3.5 & 100 & 0.0565 \\
\hline 2 & 5.5 & 50 & 0.0106 \\
\hline 3 & 5.5 & 100 & 0.0251 \\
\hline 4 & 5.5 & 200 & 0.0497 \\
\hline 5 & 8.0 & 100 & 0,0128 \\
\hline
\end{tabular}

Table 1: The operating conditions considered in this study

At the walls the boundary conditions are set as no-slip for the gas phase and partial-slip, using either the Johnson and Jackson or Schneiderbauer formulation, for the particle phase. 


\subsection{Material properties}

Air at room temperature is specified as the fluidising gas. The solid phase consists of FCC catalyst particles with a density of $1500 \mathrm{~kg} / \mathrm{m}^{3}$ and a mean diameter of $67 \mu \mathrm{m}$, as specified in the experimental work [30, 31].

\subsection{Data collection}

All simulations are performed initially for $7.5 \mathrm{~s}$ to allow for a statistical steady state to be reached, which is determined from the average mass flux in the system. A further 20s of simulated time is performed subsequently to allow important flow variables to be timeaveraged. For Case 5 the results are time-average over 50s, since it requires more time to yield symmetrical results. Time-averaged values are calculated for the solids flux and for particle velocity and volume fraction profiles. These values are compared with experimental data. The particle velocities and volume fractions are further averaged over the length of the period section. The solids velocities are calculated as a mass weighted average, since the measuring technique employed in the experiments will tend to more heavily weigh the velocities in dense regions [33]. Although no experimental data is available for it, the timeaveraged granular temperature profile is also determined, since it is an important quantity in explaining the simulation behaviour during this study.

\section{Results and Discussion}

\subsection{Comparison of the Johnson and Jackson and Schneiderbauer models}

As a start to the investigation, the performance of the Schneiderbauer model and the Johnson and Jackson model with two different specularity coefficients is investigated over a large range of flow conditions, as summarised in Table 1 . The simulations using the Schneiderbauer model is performed using the following values of the friction parameters: $\mu_{w}=0.5, e_{w}=0.9$ and $\beta_{0}=0.4$. These are the values that Schneiderbauer [23] found to be the optimum in their study considering a spouted bed. Since the spouted bed was operated using glass spheres with a diameter of $3 \mathrm{~mm}$, compared to the $67 \mu \mathrm{m}$ FCC catalyst particles in this study, it is expected that the performance of the model could still be improved significantly from this starting point by optimising the values for this particular case.

Two cases will be considered for the Johnson and Jackson model, corresponding to the two different approaches often used in literature. For the low friction approach, a specularity coefficient of 0.001 is specified. For the high friction approach, the specularity coefficient is calculated in such a way that the shear stress will be similar to that of the Schneiderbauer model settings used in this section for the limit of all non-sliding collisions (when the value of the normalised slip velocity, $\overline{v_{s}}$, in Equation 11 is low). This criterion can be calculated by setting the shear stress in Equation 6 and Equation 8 equal and using the knowledge that at low values of $\overline{v_{s}}$ the gradient of the error function is constant and therefore $\operatorname{erf}\left(\overline{v_{s}}\right)=\frac{2}{\sqrt{\pi}} \overline{v_{s}}$. This holds to within $5 \%$ for $\overline{v_{s}}<\approx 0.275$ and yields the condition that 
$\phi=\frac{6 \sqrt{2} \varepsilon_{s, \max }}{7 \pi \sqrt{3 \pi}}\left(1+\beta_{0}\right)$

giving $\phi=0.111$ for this study, using a maximum packing of 0.63 . A particle-wall restitution coefficient of 0.9 was used for both of the Johnson and Jackson approaches.

The results for the conditions considered are summarised in Figure 1. Plots are shown for the time-averaged values of the solids y-velocity and the solids volume fraction for each case, where experimental data are available. An additional plot is also shown for the granular temperature, since the granular temperature helps to explain some of the behaviour observed. It is assumed that the experimental data, given as a function of the radius, is completely symmetric, since no mention of any asymmetry is made [30, 31]. 
Particle y-velocity (m/s)

1)

2)
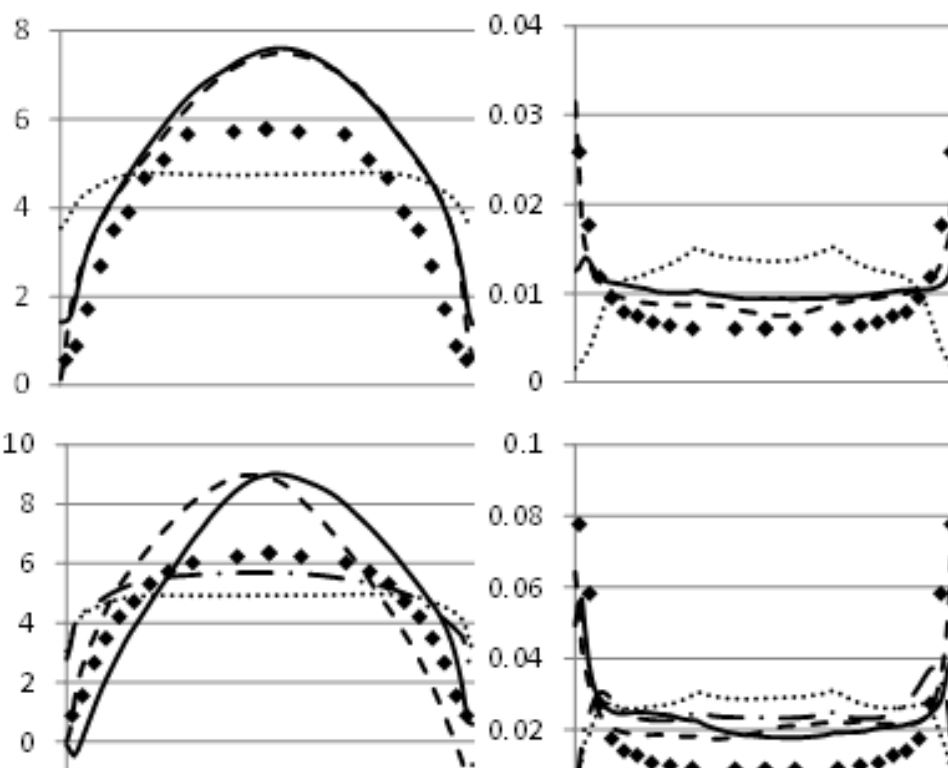

3)
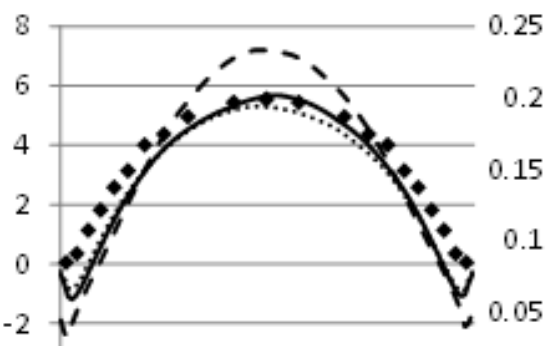

Particle volum efraction

Granular tem perature $\left(\mathrm{m}^{2} / \mathrm{s}^{2}\right)$
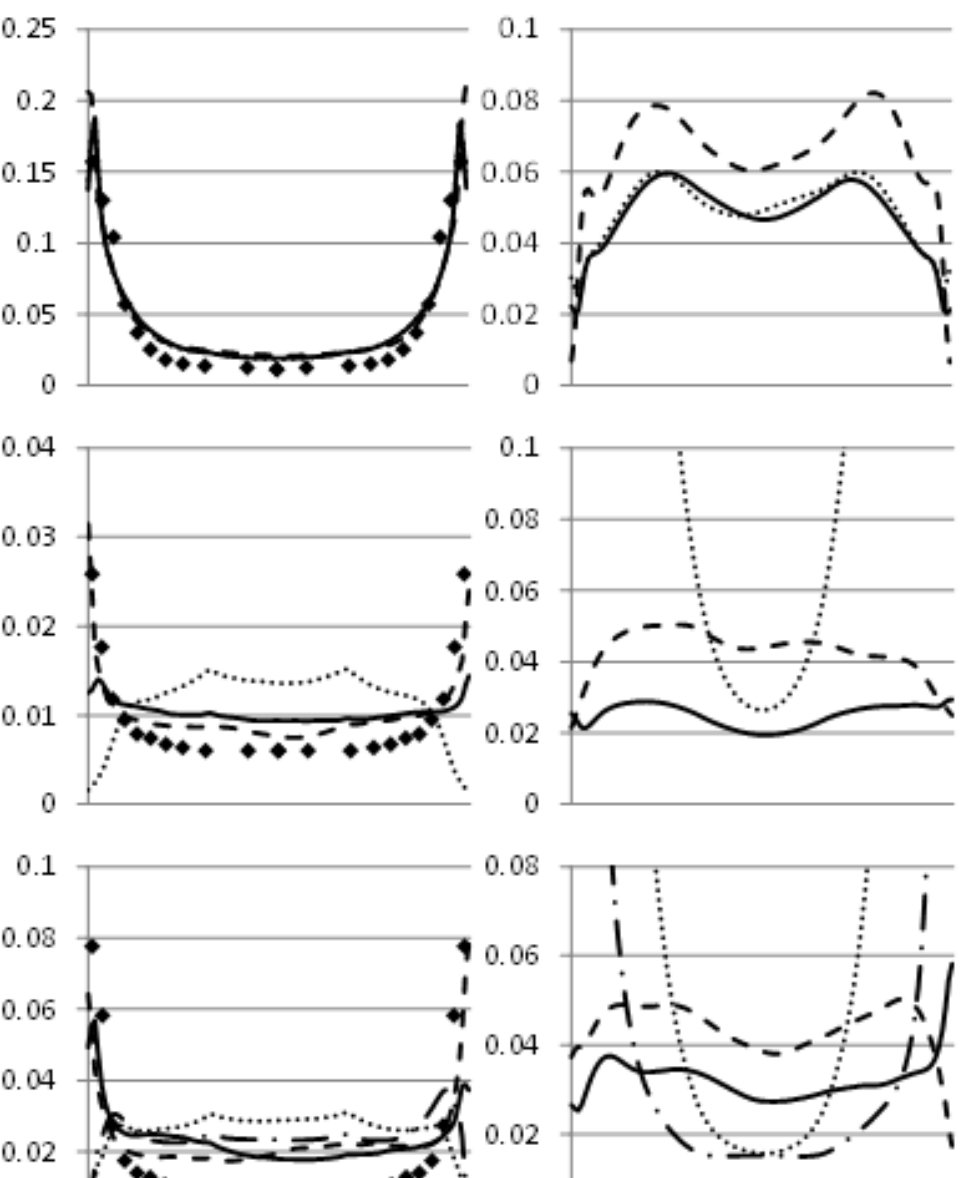

I.

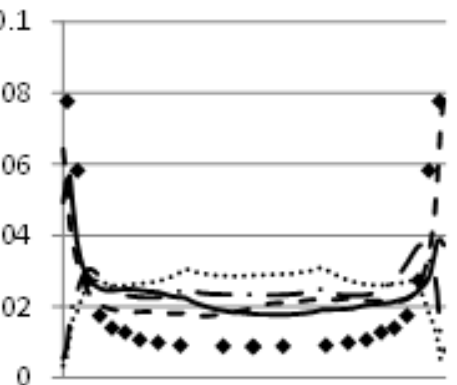

.08

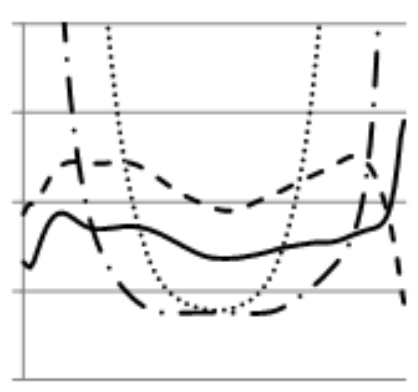

0

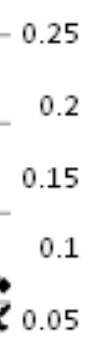

0.05

4)
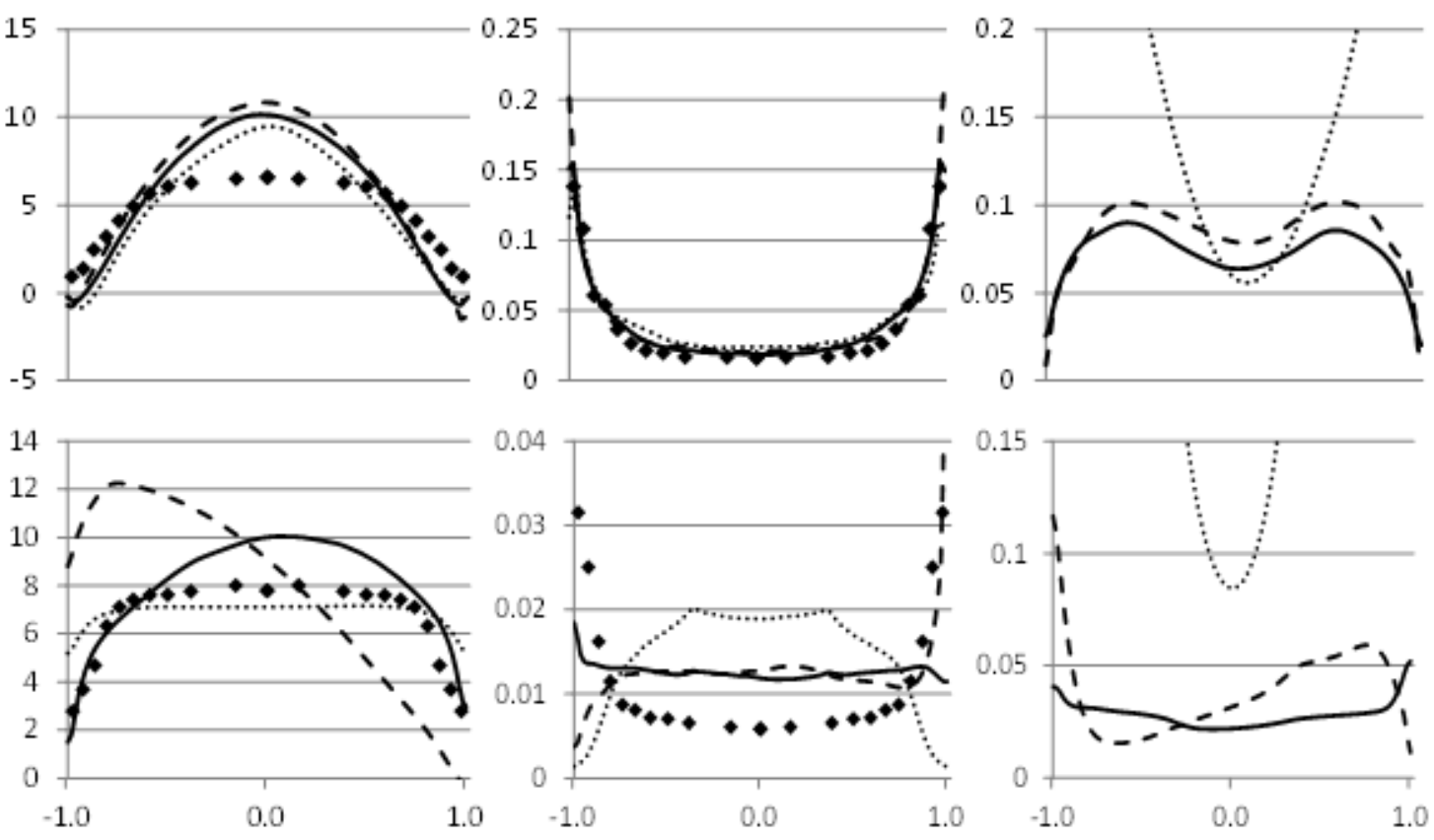

Figure 1 - Results comparing the solids y-velocity, particle phase volume fraction and granular temperature for simulations and experiments. Experiments $(\bullet \bullet)$, Schneiderbauer model ( $\longrightarrow$ ), Johnson and Jackson with $\phi=0.111$ (…), Johnson and Jackson with $\phi=0.001$ (- - ), Johnson and Jackson with $\phi=0.111$ and $e_{w}=0.2(-\cdots-)$ 


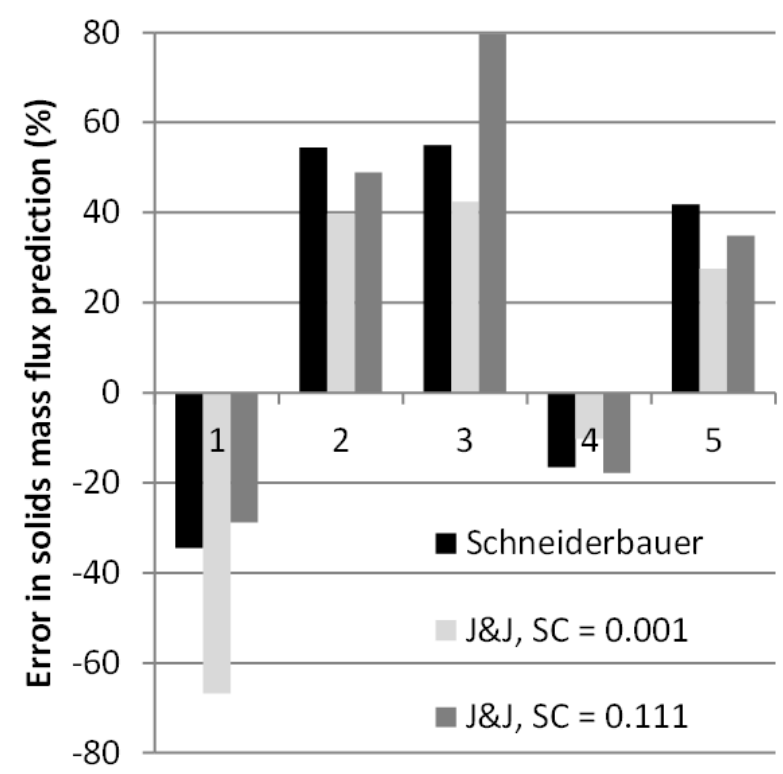

Figure 2 - Error in the solids mass flux prediction for each of the wall friction modelling methods

These results will now be discussed in a sequential manner.

\subsubsection{Case 1}

Considering the solids velocity and volume fraction for Case 1 in Figure 1 it can immediately be observed that the Schneiderbauer model and the Johnson and Jackson model at the nonsliding limit $(\phi=0.111)$ give very similar results. The reason is that, in the dense case with low gas velocity, large, slow-moving clusters form at the wall, shielding the wall from the fast moving particles. Most of the solids at the wall subsequently have a low slip velocity, leading to non-sliding collisions. The agreement of both methods with the experimental data is good, except for a small over-prediction in the downwards solids flux at the walls, leading to an under-prediction in the solids flux, shown in Figure 2. For the simulation with the low specularity coefficient the low friction at the walls often leads to thin streaks of solids rapidly falling at the wall. This causes a slightly larger overestimation of the downward solids flux at the wall and a substantial increase in the under-prediction of the solids flux (67\%, compared to $34 \%$ for the Schneiderbauer model). The flow behaviour described for Case 1 is shown in Video 1 under statistically steady state operation. 


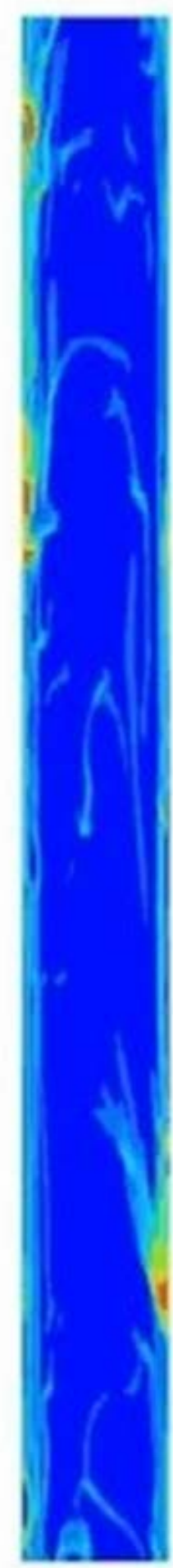

\section{J\&J}

No sliding limit
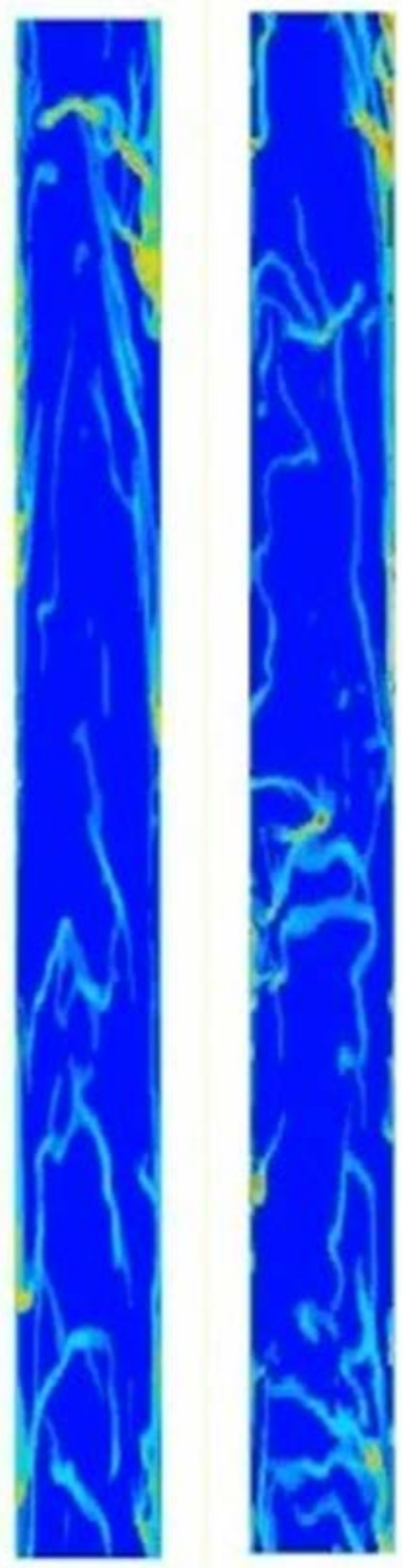

\section{J\&J Schneiderbauer \\ 0.001}




\subsubsection{Cases 2 and 3}

The conclusions that can be drawn for Cases 2 and 3, where faster, more dilute flows occur, are similar. Here, the Johnson and Jackson model with a high specularity coefficient fails completely, predicting much too high upward solids velocities and very low solids volume fractions at the walls. This indicates that there are essentially no solids clusters forming at the walls. By examining the granular temperature profiles, the cause can be seen to be very large granular temperatures at the walls. Since the flow is fast, a high shear stress at the wall causes large velocity gradients in the wall region. These large gradients give rise to large generation of granular temperature (first term on the RHS of Equation 5) which creates higher granular viscosities. The resulting highly viscous flow causes even more granular temperature generation in Equation 5 and also forces more slip at the wall despite the high degree of wall shear stress, thus resulting in a significant positive granular temperature flux at the wall according to Equation 7. To separate the effects of Equation 5 and Equation 7, an additional simulation was performed using a specularity coefficient of 0.111 and a very low restitution coefficient of 0.2 in Case 3 to test whether the problem with large granular temperatures at the wall can be solved by substantially increasing the dissipation term in Equation 7. However, results in Figure 1 show that despite a small improvement, the effect is not sufficient to deliver good results. Additionally, such nearly completely inelastic collisions are not physically realistic for the FCC catalyst particles considered in this study.

This problem with the Johnson and Jackson model is overcome by using a very low specularity coefficient value, such as 0.001 . In such a case the self-strengthening mechanism for granular temperature generation described above is avoided, allowing clusters to form. It can be observed from Figure 1 that this approach yields a very good comparison with experimental data for the particle velocity and volume fraction close to the walls. However, the deviations are observed at the centre of the riser. In the experiments the peak velocity is significantly lower and the profile much more flat. The volume fraction in the centre is also over-predicted in the simulations. Similar results occur for the Schneiderbauer model and at most of the operating conditions. Therefore it is likely that these errors are due to the $2 \mathrm{D}$ domain discretization employed, limitations of the KTGF closures used or the periodic assumption, and not due to the wall friction models. For this reason this discussion will generally focus more on the comparison with experimental data close to the walls.

The solids velocities estimated by the Schneiderbauer model are similar to that of the low friction Johnson and Jackson simulations. However, lower volume fractions are obtained at the walls, indicating that the current setup underestimates the cluster formation at the walls. The granular temperature plots for Case 2 and 3 shows a slight increase in the granular temperature close to the walls. This suggests that the generation of granular temperature at walls might be too high with the current friction parameters, deterring cluster formation.

\subsubsection{Case 4}

In Case 4 all three approaches perform reasonably well. Therefore, this denser case somehow allows the formation of clusters in the case of the Johnson and Jackson model with a 
specularity coefficient of 0.111 , despite the high granular temperature still observed for this approach in Figure 1. By observing videos of the flow for this case it was determined that for this approach cluster formation is much slower than for the other two. During start up solids clusters form in the centre of the riser, but are not allowed to form at the walls. Eventually, these clusters become sufficiently dense to overcome the excessive granular pressure gradient and reach the walls where they are maintained in a manner similar to Case 1 . The timeaveraged granular temperature remains excessively high due contributions from dilute wall regions (dense and dilute regions are weighed equally in the averaging procedure).

For all three approaches in Case 4 the upward particle velocity at the centre and the downward solids flow at walls are overestimated, but otherwise the general comparison is good. All three methods yield exceptional comparisons with the experimental data for the particle volume fraction. Figure 2 shows that all three methods slightly under-predicts the average solids flux through the domain, with the low friction Johnson and Jackson method performing slightly better than the other two methods.

\subsubsection{Case 5}

The flow behaviour in Case 5 is shown in Video 2. It is clear that the large friction Johnson and Jackson approach fails in a similar way as was described for Cases 2 and 3. For the other two methods highly asymmetric flow can be observed, which is characteristic of all instances of Case 5 considered in this study. Clusters will initiate at one wall first and start falling, lowering the upward gas velocity on that side. The gas flow then tends to move towards the other wall to bypass the solids and accelerates to maintain the same gas flux through the system. Due to the high gas velocity used in this case, this leads to high granular temperatures at the wall opposite to the one containing clusters, preventing clusters from forming on the this side as well. 


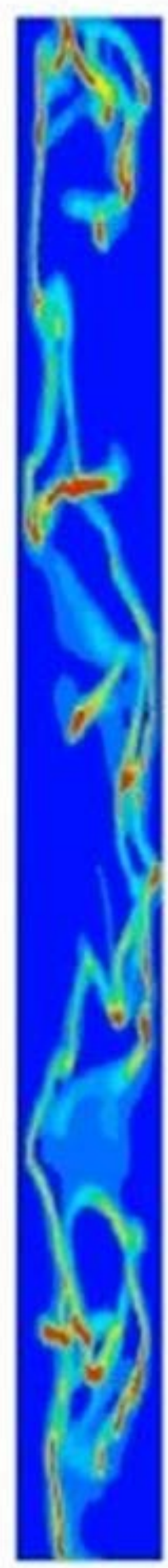

J\&J

No sliding
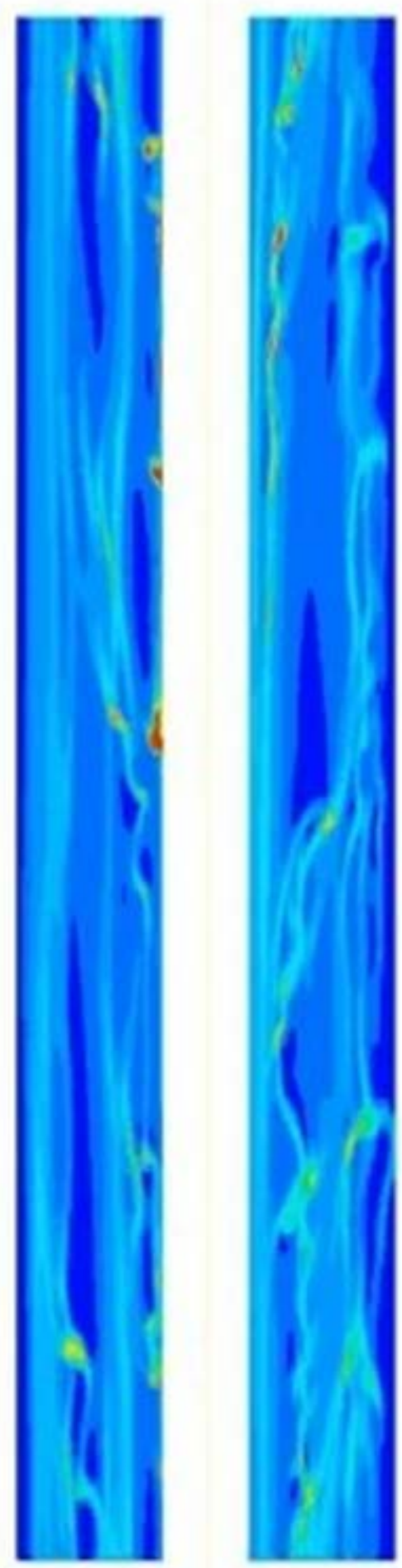

J\&J Schneiderbauer 0.001

Video 2 - Animation showing the behaviour of solids clusters in the riser section for the different wall friction models for a superficial gas velocity of $8 \mathrm{~m} / \mathrm{s}$ and a solids flux of $100 \mathrm{~kg} / \mathrm{m}^{2} \mathrm{~s}$ (Case 5). Red corresponds to a particle volume fraction of 0.1 , blue to very low solids volume fractions. The speed of the playback has been slowed down by a factor of 8 compared to the real flow time. 
However, viewing Figure 1 for Case 5, a clear difference between the Schneiderbauer model and the Johnson and Jackson model with a low specularity coefficient is evident. For the Johnson and Jackson model the particle velocity and volume fractions are completely skewed, indicating that the asymmetrical flow described above persists for the entire simulation. From Video 2 it can be seen that the reason for this is that, when using the Johnson and Jackson model with a specularity coefficient of 0.001 , every time a cluster starts growing a part of it is sheared off before it can become large enough to be swept from the wall completely. This is because of the very large shear caused by the rapidly falling clusters in this near free-slip condition.

For the Schneiderbauer model there is little cluster formation occurring, most likely due to large granular temperature at the walls. However, when it forms, clusters on one wall can break up completely, allowing a chance for clusters to be formed at the opposite side. If enough time is allowed for averaging the results, periods with clusters at each wall will cancel each other out, leading to more symmetrical results in the profile. Despite the more symmetric averaged profile for the Schneiderbauer model, the comparison with the experimental solids volume fractions is still relatively poor. The simulation predicts much higher solids volume fractions at the centre, while failing to predict enough cluster formation next to the walls.

\subsubsection{Summary of initial results}

From the results discussed in this section it can be concluded that there are two different regimes for solids flows next to the walls, both of which should be treated correctly. The first is rapid, dilute flows occurring before the onset of cluster formation. In this regime, it is important that wall shear stresses should be sufficiently small to prevent excessive strain rates in the wall regions which leads to unphysical self-strengthening granular temperature generation. This confirms the conclusion by Benyahia [17] that a specularity coefficient close to free-slip should be used to limit the granular temperature generation at the walls.

The second regime occurs when solids clusters have already formed at the wall. Here dense clusters will fall under the influence of gravity and it becomes important that sufficient wall shear stresses are included to limit the slip velocity of these dense clusters next to the wall. If the flow is sufficiently dense, the Johnson and Jackson model with a high specularity coefficient correctly models this regime and the dense clusters shield the wall from high velocity flows, thereby preventing excessive granular temperatures in dilute regions as described above.

The promise of the Schneiderbauer model therefore lies therein that it can treat both regimes accurately. By accounting for sliding collisions at large slip velocities the granular temperature generation should be limited enough to allow cluster formation at the walls, while realistic shear stresses at the walls are retained for clusters falling relatively slowly at the walls. It therefore has the best potential to give reasonable results over the entire wide range of flow conditions considered in this study with a single set of model parameters. 
However, since the cluster formation at the walls with the Schneiderbauer model is insufficient in Cases 2, 3 and 5, it appears as if the granular temperature generation is still too large with the settings used in this section. The rest of the study will therefore aim to optimise the friction parameters used in the Schneiderbauer model and to evaluate the performance of the optimised model.

\subsection{Attempted optimisation of the Schneiderbauer model}

Although the Schneiderbauer model parameters have the advantage of being measurable properties, they will still mostly be obtained by fitting simulation results to experimental results. This is because experimental measurements for particle-wall collision properties, in studies such as [34-36], are limited and most often reliable data will not be available for the specific material and particle size under investigation. This is especially the case for smaller particles sizes, as in this study, due to the practical difficulties of measuring collision properties for small particles. For this reason, a large number of simulations will be performed to determine the combination of friction parameters that will yield the best comparison with experimental results over all the flow conditions considered.

\subsubsection{Theoretical considerations}

To do this in a logical and structured manner, it is best to first obtain an understanding of how the Schneiderbauer model behaves with changing friction parameters. This is achieved by plotting the shear stress and the normalised granular flux $\left(q_{s} / \sqrt{3 \Theta_{s} N^{2}}\right)$ against a dimensionless slip velocity, $r=\left\|\vec{v}_{s,}\right\| / \sqrt{3 \Theta_{s}}$. The values of the three friction parameters are varied around those considered earlier in this study to gain a better understanding of their influence. Additionally, the following equations are derived for the shear stress at the limits of all non-sliding $\left(\operatorname{erf}\left(\overline{\bar{v}_{s}}\right)=\frac{2}{\sqrt{\pi}} \overline{v_{s}}\right)$ and all sliding collisions $\left(\operatorname{erf}\left(\overline{v_{s}}\right)=1\right)$ :

$$
\begin{aligned}
& \left\|\vec{\tau}_{s}\right\|=-\frac{\sqrt{2}}{7 \sqrt{\pi}}\left(1+\beta_{0}\right) \alpha_{s} \rho_{s} g_{0} \sqrt{\Theta_{s}}\left\|\vec{v}_{s, l}\right\| \\
& \left\|\vec{\tau}_{s}\right\|=-\frac{1}{2}\left(1+e_{w}\right) \mu_{w} \alpha_{s} \rho_{s} g_{0} \Theta_{s}
\end{aligned}
$$

First considering the shear stress, it can immediately be seen from Equation 14 that the shear stress in the non-sliding regime is only dependent on the tangential restitution coefficient, while in the sliding regime, Equation 15, the shear stress is dependent on the friction coefficient and the normal restitution coefficient. This can be confirmed from the plots in Figure 3. For sliding collisions the shear stress is independent of the slip velocity and its value decreases with a reduction in either the friction coefficient or the normal restitution coefficient. For non-sliding collisions the shear stress is directly proportional to the tangential slip velocity. It is also clear that the friction coefficient has a larger influence on the shear stress than the restitution coefficients, due to the restitution coefficients occurring in the form $\left(1+e_{w}\right)$. The friction parameters also influence the onset of sliding collisions. The friction coefficient again has the largest influence with sliding collisions starting earlier at low friction coefficient values. 
a)
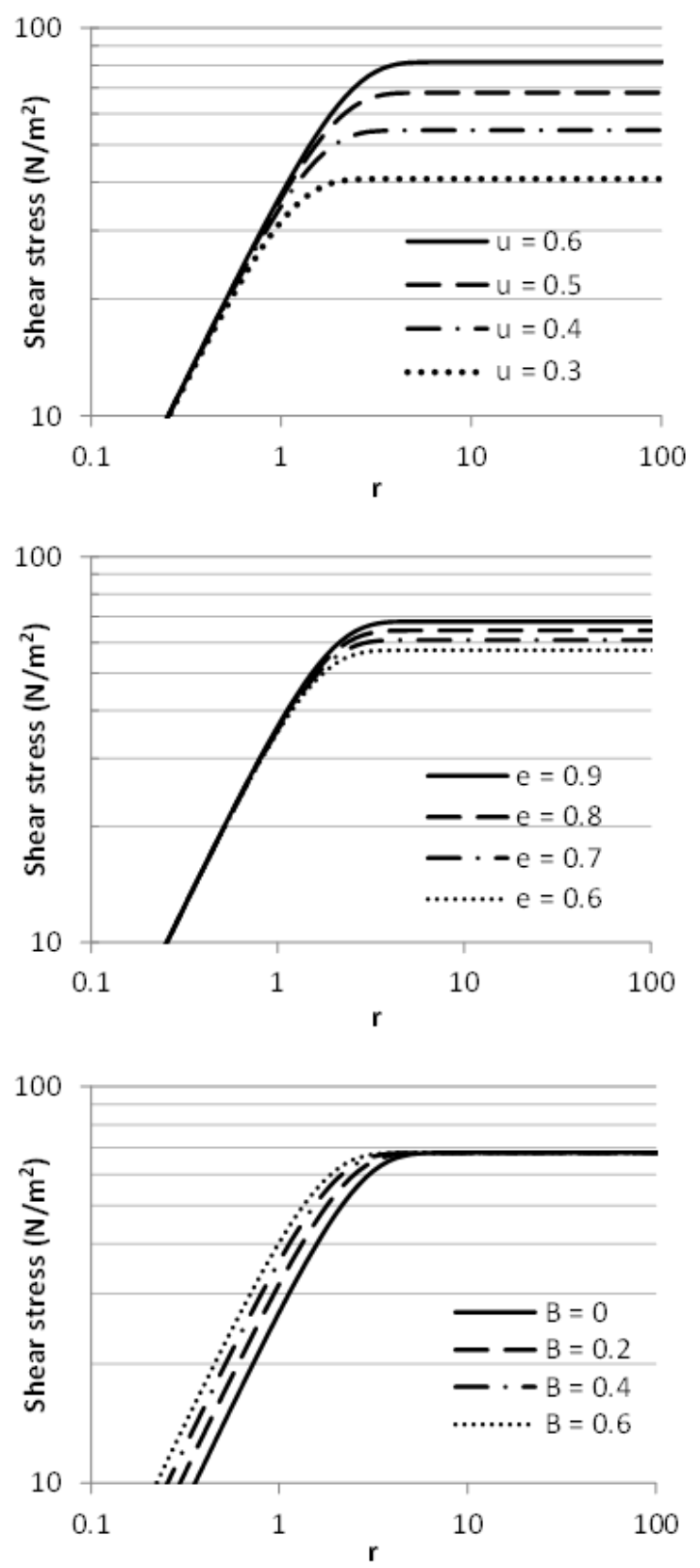

b)
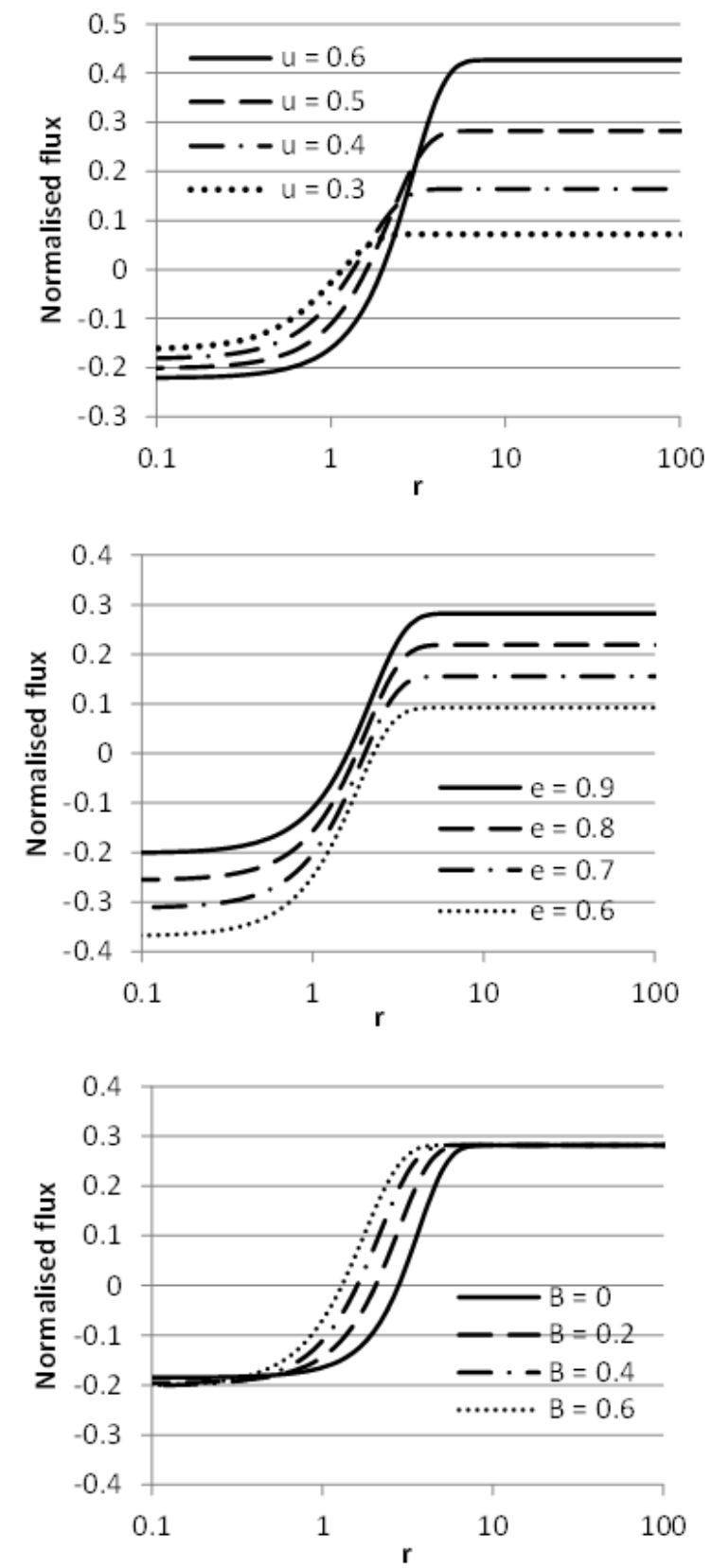

Figure 3 - Plot of a) the shear stress and b) the normalised flux, $q_{s} / \sqrt{3 \Theta_{s} N^{2}}$, plotted against the non-dimensional slip velocity, $r=\left\|\vec{v}_{s, \|}\right\| / \sqrt{3 \Theta_{s}}$. The effects of changes in the friction coefficient, normal restitution coefficient and tangential restitution coefficient are shown separately.

Due to the complexity of the granular flux equation, the behaviour of the granular flux cannot that easily be deduced from simplified equations. However, by plotting the behaviour of the granular temperature flux with changing values of the friction parameters, several important observations can be made. Firstly, the friction coefficient is once again the most influential. Increasing its value both increases the positive granular temperature flux in the sliding regime and the negative flux in the non-sliding regime. The normal restitution coefficient has a smaller, but more regular, effect. By specifying more elastic collisions the granular 
temperature flux to the solids increases. Lastly, the tangential restitution coefficient has the smallest influence on the granular temperature flux, with an increase in its value primarily allowing sliding collisions to start at lower velocities.

Since the number of simulations required to test the influence of all three parameters on all 5 cases would be impractically large, the sensitivity analysis will be carried out only for the two most influential parameters and two most interesting cases. Regarding the parameters, the tangential restitution coefficient is not considered in this optimisation study. Results in section 3.1 showed the primary shortcoming of the current setting in the Schneiderbauer model is its ability to predict cluster formation in fast, dilute flows. Excluding the tangential restitution coefficient is therefore reasonable, since the tangential restitution coefficient has no influence on the granular flux in the sliding regime. The current value of 0.4 delivered good results in the denser cases, where mostly non-sliding collisions occur at the wall and the tangential restitution coefficient will therefore be most influential, and therefore appears to be an appropriate choice. Consequently, it was decided to run simulations for all combinations of $\beta_{0}=0.4, \mu_{w}=0.5,0.4,0.3$ and $e_{w}=0.9,0.7,0.5$. Lower values of the parameters were explored, since in both cases this will lead to less granular temperature generation at high slip velocities.

Regarding the cases, it was decided to focus on Cases 1 and 5 because they represent the outer bounds of the range of conditions considered in this study.

\subsubsection{Simulation results}

To allow for easier interpretation of results, the profiles will from now on be displayed averaged over the plane of symmetry. From Figure 1 is can be seen that the results for all cases, except Case 5, is highly symmetric and this approach is therefore justified. Figure 4 shows that, given enough averaging time, the profile for Case 5 will also become symmetric when using the Schneiderbauer model. To maintain practical computational times, simulations of Case 5 are therefore run for 50s, after which the profiles are averaged over the symmetry plane.

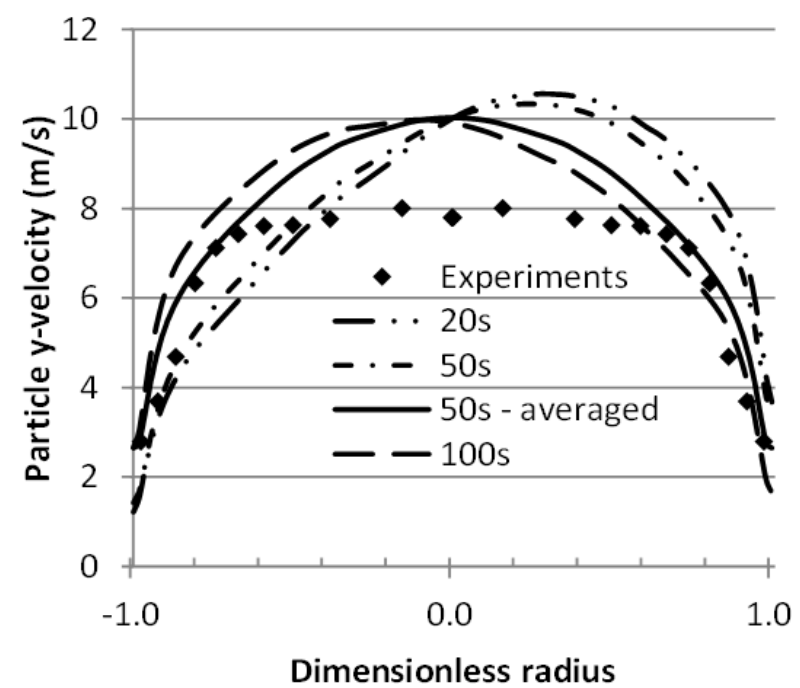


The effect of changing the friction parameters in Case 5 can best be seen from the timeaveraged solids volume fraction profiles shown in Figure 5, since the solids velocity profile proved to be insensitive to changes in the wall boundary conditions. It is evident that the solids volume fraction at the wall increases as the value of either the friction coefficient or the normal restitution coefficient is lowered. This is a result of less granular temperature generation at the walls producing the desired effect of more clusters forming at the walls. However, even at very low friction values the simulations are unable to reproduce the high solids volume fraction at the walls or the low volume fraction at the riser centre. 


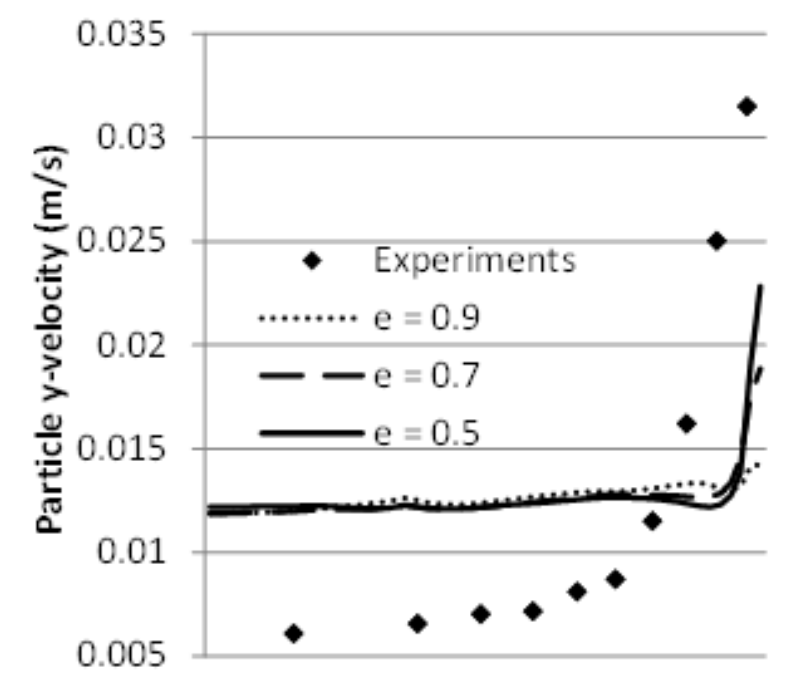

a)

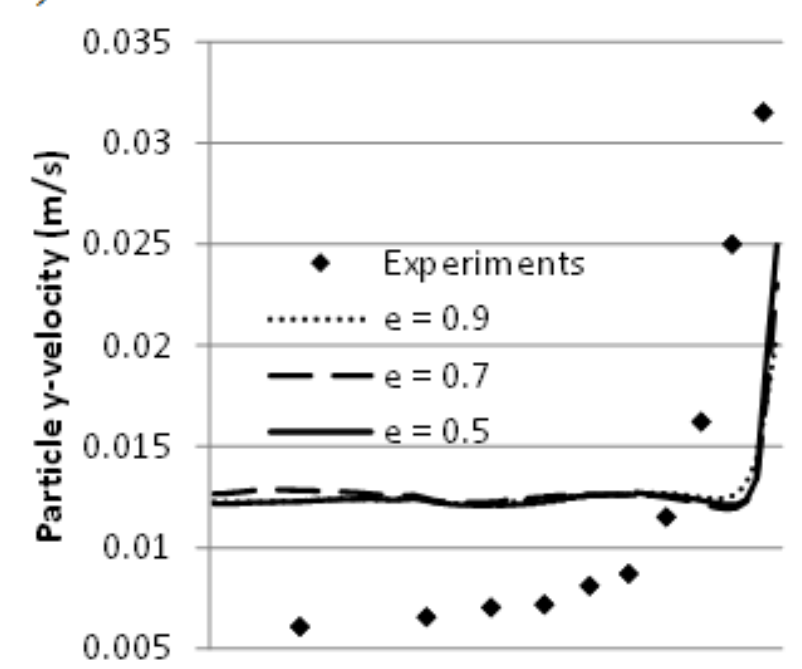

b)

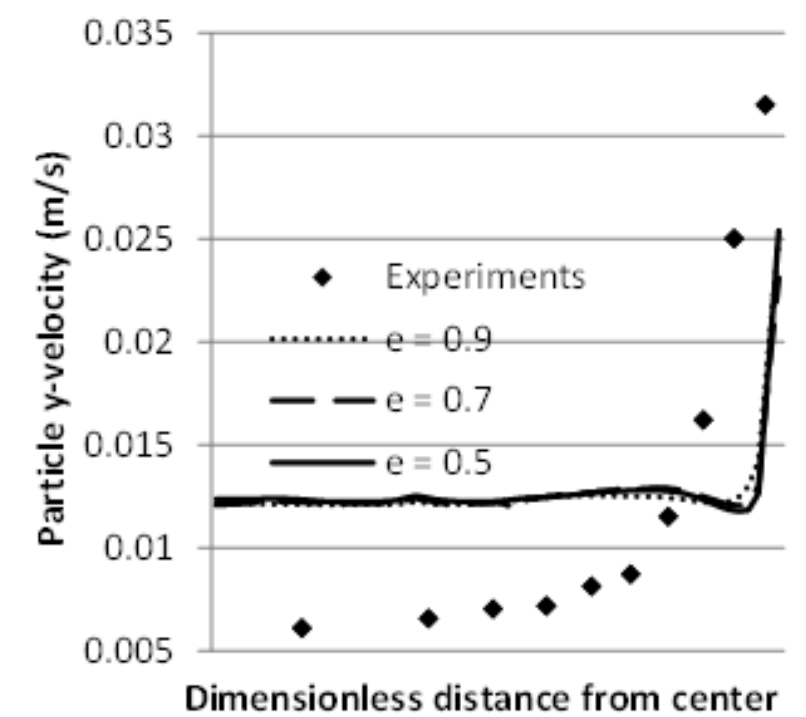

c)

Figure 5 - Time-averaged particle volume fraction profiles for Case 5 using the Schneiderbauer model at different normal restitution coefficients for friction coefficient values of a) $0.5, \mathrm{~b}) \mathbf{0 . 4}$ and c) 0.3 
For Case 1 it was established that for these conditions the time-averaged volume fraction is insensitive to changes in the wall boundary conditions, therefore the solids y-velocity is shown in Figure 6. Here it is shown that, at the highest friction coefficient investigated ( $\mu_{w}=0.5$ ), a small reduction in solids velocity next to the wall is seen when lowering the normal restitution coefficient to a value of 0.5 . This seemingly small effect created a considerable increase in the underestimation of the solids flux (39\% to 67\% by changing $e_{w}$ from 0.7 to 0.5 ), shown in Figure 7 . At lower friction coefficients it can be seen that, by further decreasing the normal restitution coefficient, the simulations strongly over-predict down-flow at the walls. This leads to even greater under-predictions of the solids flux and even negative fluxes in the worst scenarios. To maintain the same gas flow rate through the system, the gas in the centre accelerates, leading to a considerable over-prediction of the upwards solids velocity in the centre.

This significant change in behaviour is because combinations of sufficiently low values for the friction coefficient and normal restitution coefficient lead to the onset of sliding collisions at lower velocities. This causes clusters falling at the walls (normally considered to be in the non-sliding regime) to enter the sliding regime, where the shear stress is highly dependent on the friction coefficient and the normal restitution coefficient. In this way the shear stress on solids clusters at the walls is reduced, resulting in the large downward flux of solids that is observed 


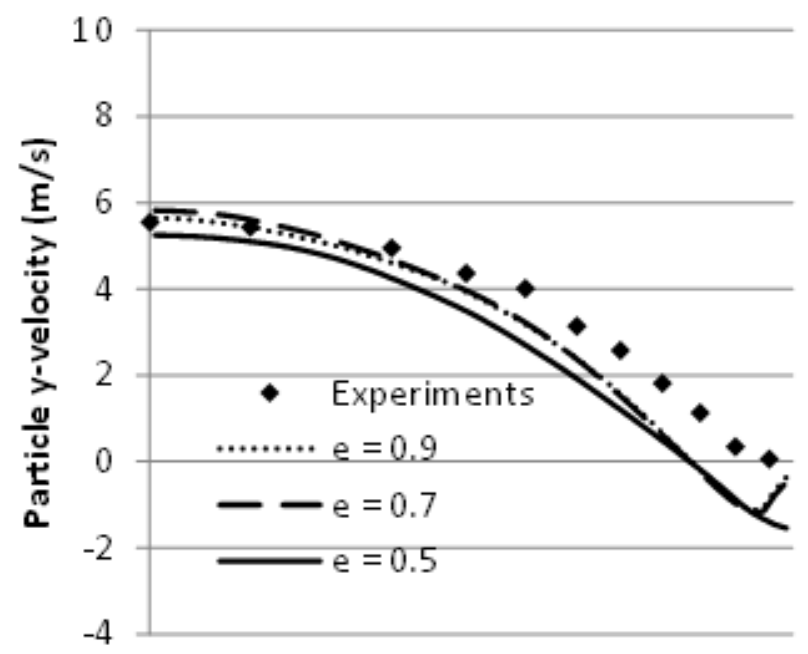

a)

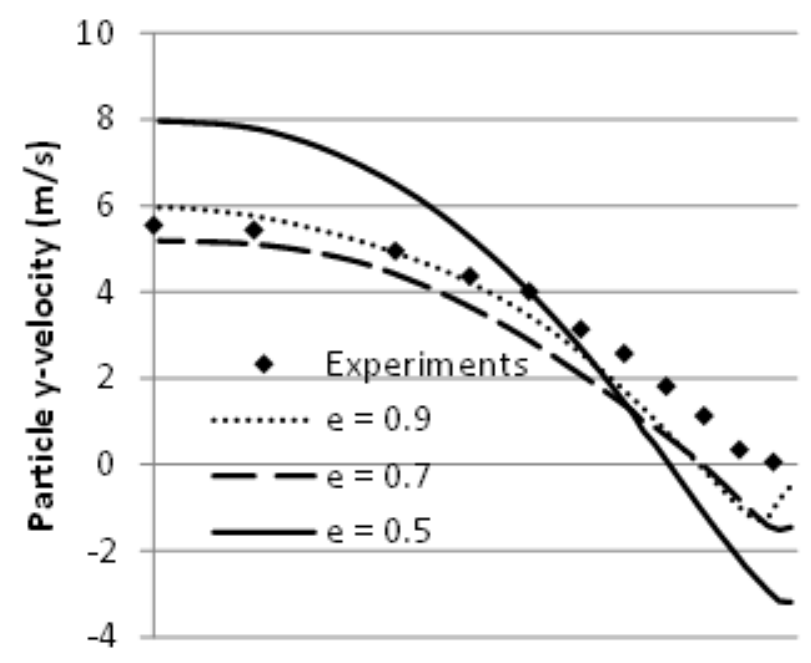

b)

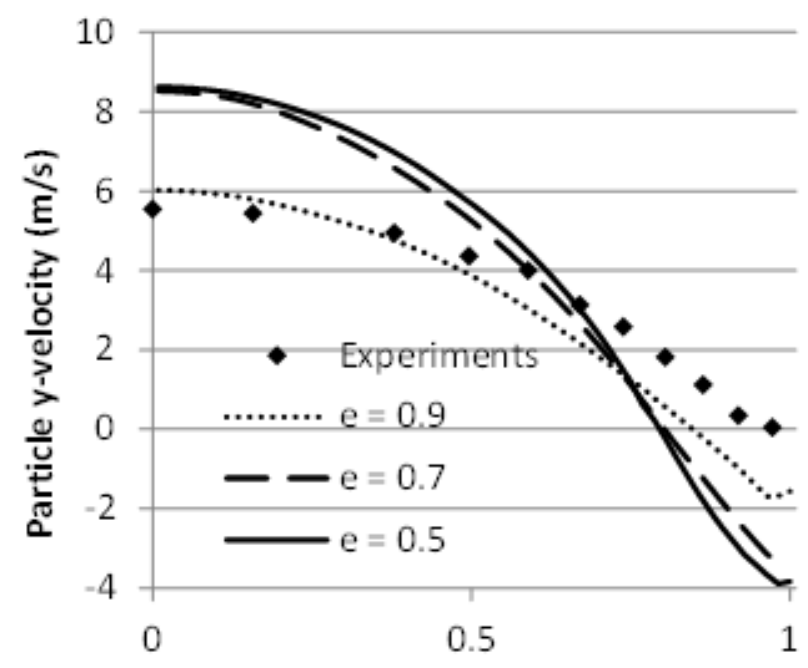

Dimensionless distance from center

c)

Figure 6 - Time-averaged particle y-velocity profiles for Case 1 using the Schneiderbauer model at different normal restitution coefficients for friction coefficient values of a) 0.5 , b) 0.4 and c) 0.3 


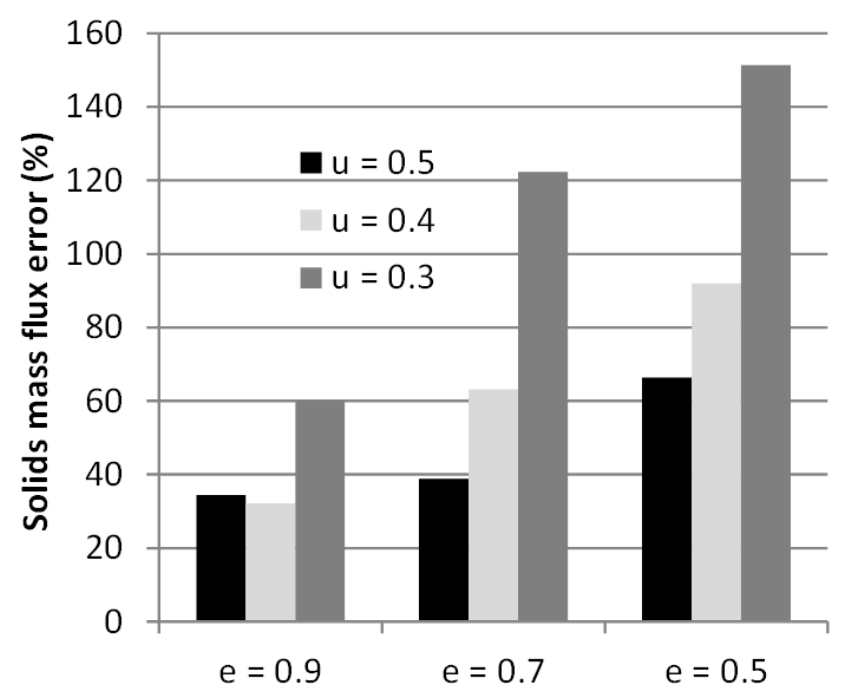

Figure 7 - Comparison of the percentage under-prediction in the solids mass flux compared to experimental data for Case 1 when performing simulations using the Schneiderbauer model and different combinations of values for the friction coefficient and the normal restitution coefficient.

It can therefore be concluded that by lowering the values of the two parameters considered, the granular temperature generation is decreased and more clusters will form, lessening this shortcoming described in section 3.1. However, this also decreases the shear stress at the walls, which can lead to too large downward solids flows at the walls. To obtain the optimum setting for the Schneiderbauer model in this study the settings that greatly over-predicts the downward flow of the solids at the walls in the dense case (Case 1) were first eliminated. This leaves the following settings as options: $\mu_{w}=0.5, e_{w}=0.9, \mu_{w}=0.5, e_{w}=0.7$ and $\mu_{w}=0.4, e_{w}=0.9$. From these alternatives the setting was chosen that predicts the largest solids volume fraction at the wall, and therefore also the most clusters, in the fast and dilute case (Case 5). This gives the setting of $\mu_{w}=0.4, e_{w}=0.9$, with a particle volume fraction of 0.0206 at the wall. This represents only a minor change from the original setting ( $\left.\mu_{w}=0.5, e_{w}=0.9\right)$. It was therefore not possible to substantially improve the general performance of the Schneiderbauer model further through parameter tuning, although this small modification did successfully increase the solids volume fraction at the walls in the more dilute cases (Cases 2, 3 and 5 in Figure 8). 
- Experiments $\longrightarrow$ Schneiderbauer …..... Johnson \& Jackson

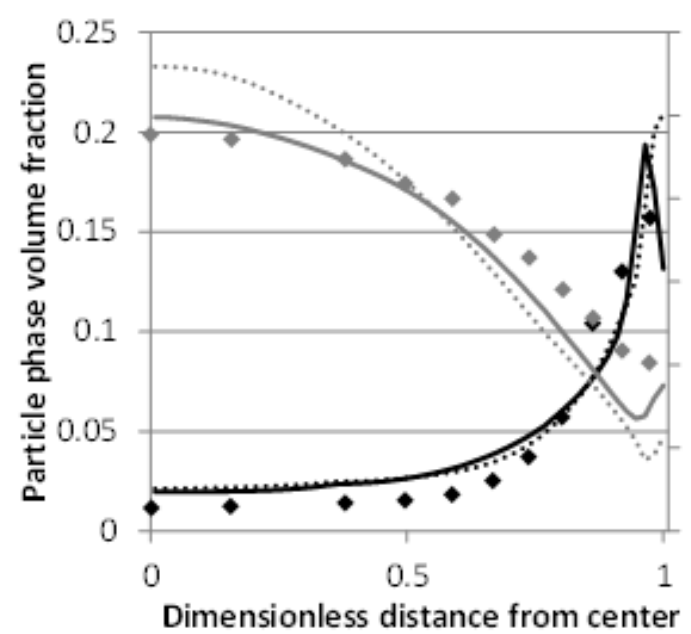

a)

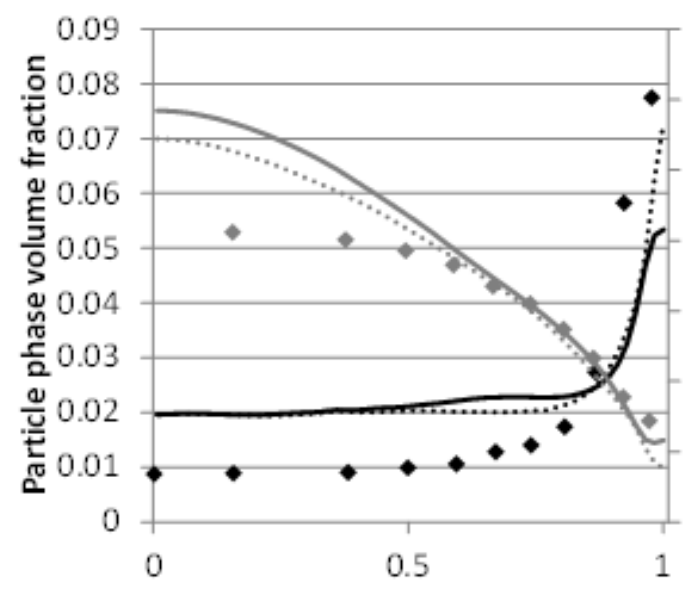

Dimensionless distance from center

c)

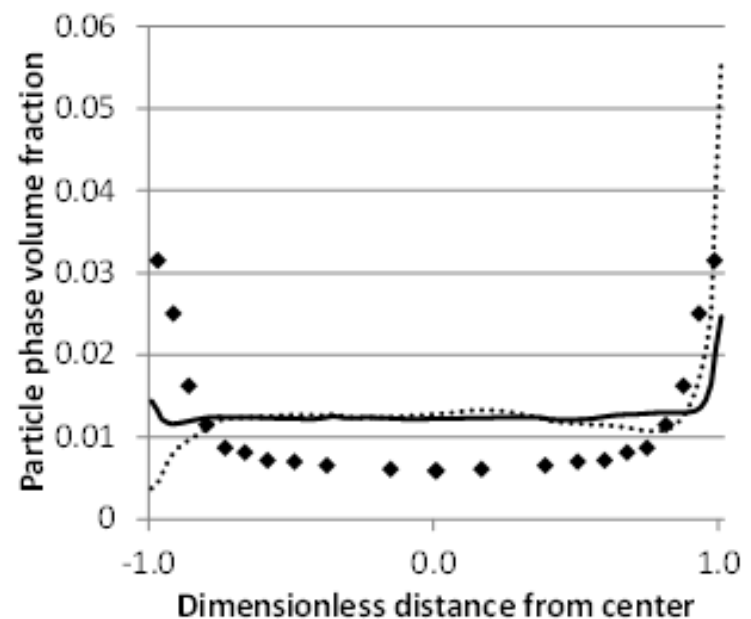

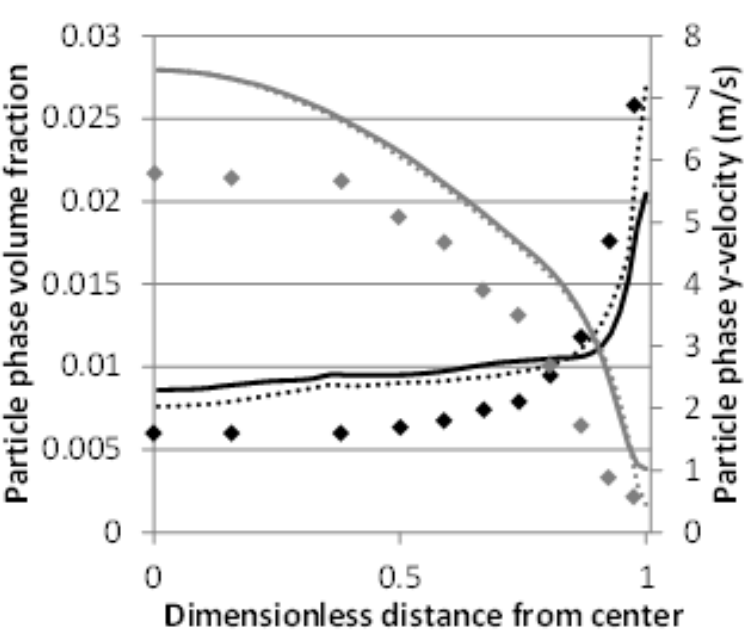

b)

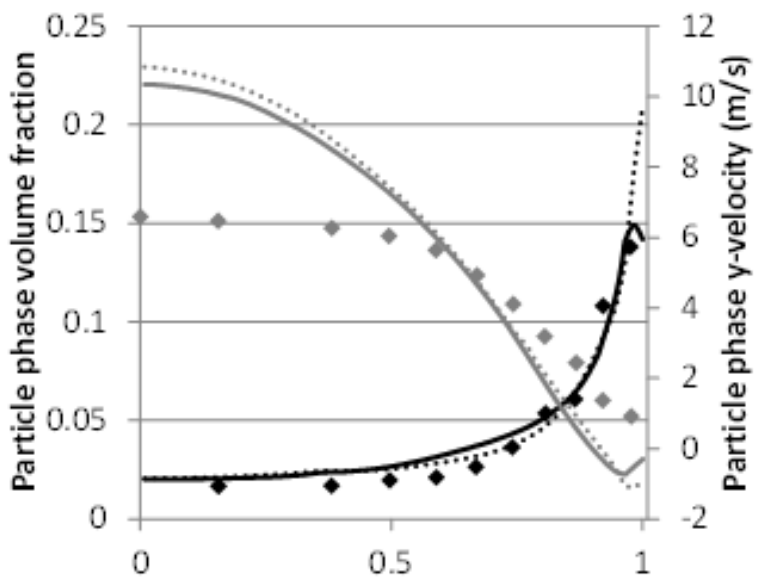

Dimensionless distance from center

d)

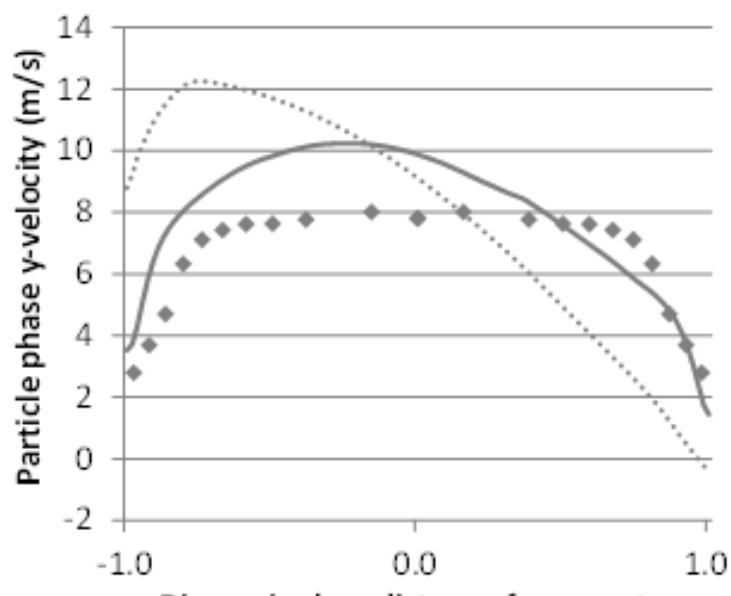

Dimensionless distance from center

e)

Figure 8 - Comparison of the performance of the optimised Schneiderbauer model to that of the Johnson and Jackson model using a specularity coefficient of 0.001 in a) Case 1, b) Case 2, c) Case 3, d) Case 4 and e) Case 5. Black is used for the time-averaged particle volume fraction, grey for the time-averaged particle $y$-velocity. 
The ultimate performance of the Schneiderbauer model over the range of cases investigated is therefore comparable to that of the near free-slip implementation of the classic Johnson and Jackson model. However, it should again be emphasized that this near free-slip implementation is not generally applicable. For example, previous work conducted by the authors in a pseudo-2D setup [37, 38] showed that high wall friction on the large front and back walls must be included to reasonably predict experimentally measured solids velocity profiles. The primary advantage of the Schneiderbauer approach is that it can deliver reasonable results in fast and dilute flows with settings which can also achieve reasonable results in slow and dense flows.

Despite returning comparable mean performance, the near free-slip Johnson and Jackson implementation resulted in significantly different dynamic behaviour to that of the Schneiderbauer model (Video 3). Clusters tend to fall much faster next to the wall and the flow is more prone to asymmetric behaviour (seemingly permanent in Case 5). As an example, the root-mean-square of the near-wall particle y-velocity in the near free-slip Johnson and Jackson implementation is $127 \%$ and $164 \%$ greater than the Schneiderbauer prediction in Cases 3 and 4 respectively.

In addition, the larger strain rates in the near free-slip Johnson and Jackson implementation also leads to substantially higher granular temperatures (Figure 1). This tends to mask the error caused by under-predicted wall shear stress by increasing the granular viscosity and thereby the resistance to further strain. A lack of shear stress on the clusters at the wall is therefore compensated for by an increase in the viscous shear force exerted by the upward flow at the centre of the geometry.

It therefore becomes clear that more detailed experimental data on the dynamic behaviour of clusters at the walls will be required to properly evaluate model performance. Such work can be strongly recommended for future study. 


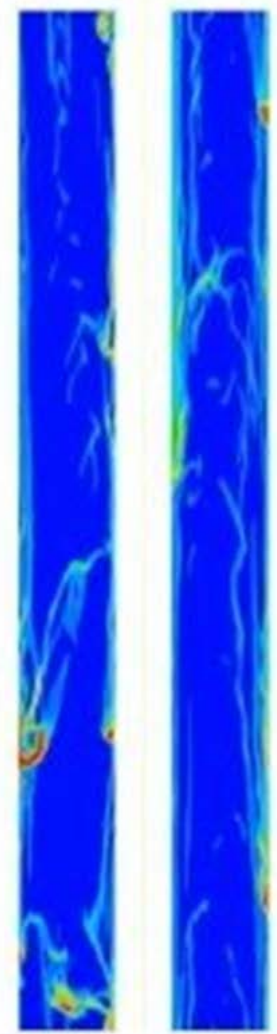

Case 1

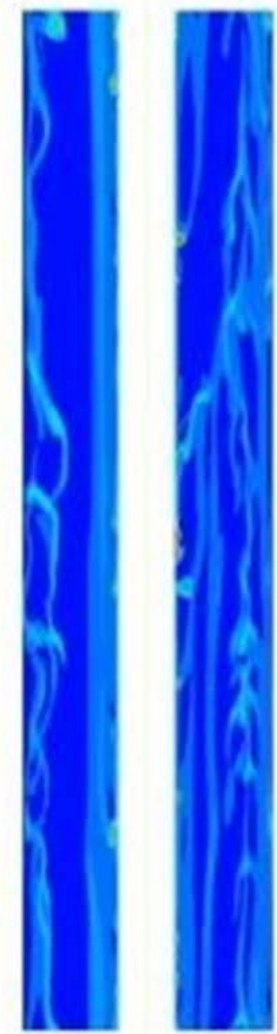

Case 2

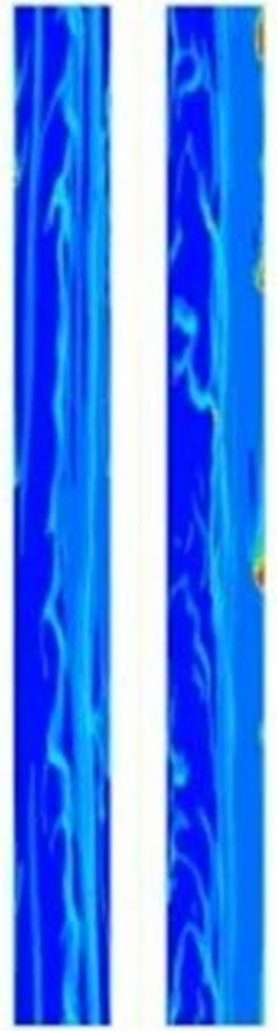

Caso 3

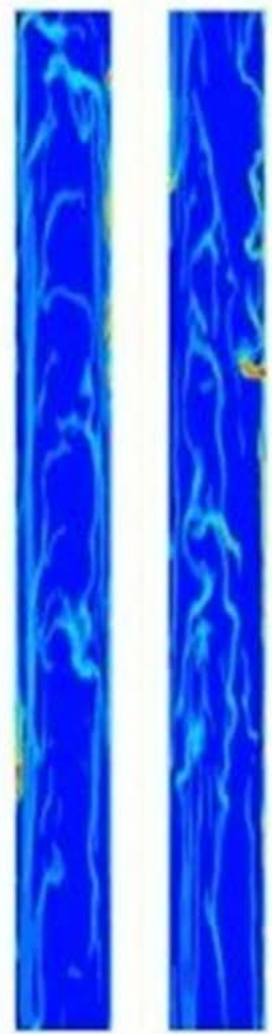

Caso 4

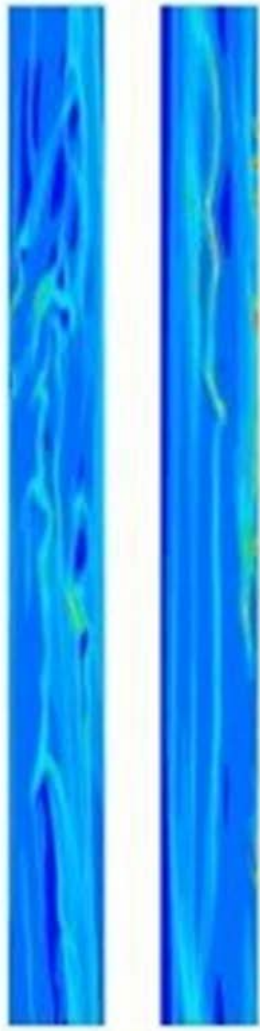

Case 5

Video 3 - Animation showing the solids volume fraction for the optimised Schneiderbauer model (left) and the Johnson and Jackson model using a specularity coefficient of 0.001 (right) for all five case considered. The maximum volume fraction (red) for each case is: Case $1=0.6$, Case $2=0.15$, Case $3=0.3$, Case $4=0.6$ and Case $5=0.1$.

\section{Summary and Conclusions}

Two formulations for the particle-wall boundary condition, the Johnson and Jackson and Schneiderbauer models, were investigated over a range of riser flow conditions in this study. The Johnson and Jackson model was tested both with reasonable partial slip settings (specularity coefficient of 0.111) and with near free-slip settings (specularity coefficient of 0.001), whereas the Schneiderbauer model was implemented with recommended coefficients. The recommended Schneiderbauer coefficients were shown to be close to optimal for the range of flow conditions considered in this study.

Results revealed that the Johnson and Jackson model with the larger specularity coefficient could only simulate denser and slower cases, failing completely in faster and more dilute cases. In such cases, this implementation of the Johnson and Jackson model strongly overpredicts the shear stress and the granular temperature generation at the walls, thus resulting in an unphysical self-strengthening generation of granular temperature in the near-wall regions.

This problem can be solved by specifying a very low specularity coefficient, as is commonly done in literature. However, this near free-slip condition is not physically reasonable and causes the Johnson and Jackson approach to perform poorly in dense flows, where the low shear stress at the walls allows solids to fall rapidly. In general, however, this approach 
performs surprisingly well when measured according to mean velocities and volume fractions. Despite this comparable mean performance, it was shown that the dynamic cluster behaviour at the walls differs significantly between the two approaches. In particular, the near free-slip Johnson and Jackson implementation is more prone to an asymmetric flow situation where clusters fall rapidly on one side of the geometry while the rising gas is pushed to the other side. More detailed experimental data on the dynamic flow behaviour will therefore be required to draw definitive conclusions on this matter and is recommended for future study.

In general, however, the Schneiderbauer model appears to offer a clear improvement over the broadly deployed classic Johnson and Jackson model. As opposed to the Johnson and Jackson model, a single set of model parameters can give reasonable results both in dense, slow moving flows and dilute, fast moving flows. Additionally, it has the benefits of using measurable properties as parameters. It can therefore be recommended for future work in simulating fluidised bed risers.

\section{Acknowledgements}

The authors would like to express their gratitude for the financial support from the European Commission under the NanoSim grant (project number: 604656). Additionally, the computational resources at NTNU provided by NOTUR, http://www.notur.no, were used during this project. The authors also thank Dr. Simon Schneiderbauer for supplying a UDF for computing the wall shear stress and the flux of granular kinetic energy at the wall.

\section{List of Symbols}

Main Symbol definitions:

$\begin{array}{ll}\alpha & \text { Volume fraction } \\ \vec{v} & \text { Velocity vector }(\mathrm{m} / \mathrm{s}) \\ \overline{\bar{\tau}} & \text { Stress tensor }(\mathrm{Pa}) \\ \phi & \text { Specularity coefficient } \\ \vec{\tau}_{s} & \text { Shear stress at the wall }\left(\mathrm{N} / \mathrm{m}^{2}\right) \\ g_{0} & \text { Radial distribution function } \\ p & \text { Pressure }(\mathrm{Pa}) \\ \overline{v_{s}} & \text { Normalised particle slip velocity } \\ e_{w} & \text { Normal restitution coefficient } \\ K_{s g} & \text { Interphase momentum exchange coefficient }\left(\mathrm{kg} / \mathrm{m}^{3} \mathrm{~s}\right) \\ \phi_{g s} & \text { Interphase energy transfer }\left(\mathrm{W} / \mathrm{m}^{3}\right) \\ \vec{g} & \text { Gravity vector }\left(\mathrm{m} / \mathrm{s}^{2}\right) \\ q_{s} & \text { Granular temperature flux at the wall }\left(\mathrm{W} / \mathrm{m}^{2}\right) \\ \Theta & \text { Granular temperature }\left(\mathrm{m}^{2} / \mathrm{s}^{2}\right)\end{array}$




$\begin{array}{ll}\mu_{w} & \text { Friction coefficient } \\ \gamma & \text { Dissipation rate }\left(\mathrm{W} / \mathrm{m}^{3}\right) \\ k & \text { Diffusion coefficient }(\mathrm{kg} / \mathrm{m} \mathrm{s}) \\ \rho & \text { Density }\left(\mathrm{kg} / \mathrm{m}^{2}\right) \\ t & \text { Time }(\mathrm{s}) \\ \beta_{0} & \text { Tangential restitution coefficient } \\ N & \text { Normal shear stress }\left(\mathrm{N} / \mathrm{m}^{2}\right)\end{array}$

Sub- and superscript definitions:

$\begin{array}{ll}g & \text { Gas } \\ s & \text { Solid } \\ w & \text { Wall } \\ \mid & \text { Slip velocity parallel to wall }\end{array}$

\section{References}

[1] S. Sundaresan, Modeling the hydrodynamics of multiphase flow reactors: Current status and challenges, AIChE J., 46 (2000) 1102-1105.

[2] D. Gidaspow, R. Bezburuah, J. Ding, Hydrodynamics of Circulating Fluidized Beds, Kinetic Theory Approach, 7th Engineering Foundation Conference on Fluidization 1992, pp. 75-82.

[3] M. Syamlal, W. Rogers, T.J. O'Brien, MFIX Documentation: Volume 1, Theory Guide., National Technical Information Service, Springfield, 1993.

[4] C.K.K. Lun, S.B. Savage, D.J. Jeffrey, N. Chepurniy, Kinetic Theories for Granular Flow: Inelastic Particles in Couette Flow and Slightly Inelastic Particles in a General Flow Field, J. Fluid Mech., 140 (1984) 223-256.

[5] Y. Igci, S. Pannala, S. Benyahia, S. Sundaresan, Validation Studies on Filtered Model Equations for Gas-Particle Flows in Risers, Ind. Eng. Chem. Res., 51 (2012) 2094-2103.

[6] C.C. Milioli, F.E. Milioli, On the accuracy of two-fluid model predictions for a particular gas-solid riser flow, Applied Mathematical Modelling, 34 (2010) 684-696.

[7] Y. Igci, S. Sundaresan, Constitutive Models for Filtered Two-Fluid Models of Fluidized Gas-Particle Flows, Ind. Eng. Chem. Res., 50 (2011) 13190-13201.

[8] S. Schneiderbauer, S. Pirker, Filtered and heterogeneity-based subgrid modifications for gas-solid drag and solid stresses in bubbling fluidized beds, AIChE J., 60 (2014) 839-854.

[9] A. Ozel, P. Fede, O. Simonin, Development of filtered Euler-Euler two-phase model for circulating fluidised bed: High resolution simulation, formulation and a priori analyses, Int. J. Multiphase Flow, 55 (2013) 43-63.

[10] S. Cloete, S. Amini, S.T. Johansen, A fine resolution parametric study on the numerical simulation of gas-solid flows in a periodic riser section, Powder Technology, 205 (2011) 103111.

[11] A. Almuttahar, F. Taghipour, Computational fluid dynamics of high density circulating fluidized bed riser: Study of modeling parameters, Powder Technol., 185 (2008) 11-23.

[12] P.C. Johnson, R. Jackson, Frictional-Collisional Constitutive Relations for Granular Materials, with Application to Plane Shearing, J. Fluid Mech., 176 (1987) 67-93. 
[13] A. Soleimani, S. Schneiderbauer, S. Pirker, A comparison for different wall-boundary conditions for kinetic theory based two-fluid models, Int. J. Multiphase Flow, 71 (2015) 9497.

[14] Y. Zhao, Y. Zhong, Y. He, H.I. Schlaberg, Boundary conditions for collisional granular flows of frictional and rotational particles at flat walls, AIChE J., 60 (2014) 4065-4075.

[15] B. Chalermsinsuwan, P. Piumsomboon, D. Gidaspow, Kinetic theory based computation of PSRI riser: Part I-Estimate of mass transfer coefficient, Chem. Eng. Sci., 64 (2009) 11951211.

[16] H. Lu, S. Wang, Y. He, J. Ding, G. Liu, Z. Hao, Numerical simulation of flow behavior of particles and clusters in riser using two granular temperatures, Powder Technol., 182 (2008) 282-293.

[17] S. Benyahia, M. Syamlal, T.J. O'Brien, Study of the ability of multiphase continuum models to predict core-annulus flow, AIChE J., 53 (2007) 2549-2568.

[18] J.T. Jenkins, Boundary Conditions for Rapid Granular Flow: Flat, Frictional Walls, Journal of Applied Mechanics, 59 (1992) 120-127.

[19] M.Y. Louge, Computer simulations of rapid granular flows of spheres interacting with a flat, frictional boundary, Physics of Fluids, 6 (1994) 2253-2269.

[20] T. Li, S. Benyahia, Revisiting Johnson and Jackson boundary conditions for granular flows, AIChE J., 58 (2012) 2058-2068.

[21] S. Schneiderbauer, D. Schellander, A. Löderer, S. Pirker, Non-steady state boundary conditions for collisional granular flows at flat frictional moving walls, Int. J. Multiphase Flow, 43 (2012) 149-156.

[22] J.T. Jenkins, M.Y. Louge, On the flux of fluctuation energy in a collisional grain flow at a flat, frictional wall, Physics of Fluids, 9 (1997) 2835-2840.

[23] S. Schneiderbauer, A. Aigner, S. Pirker, A comprehensive frictional-kinetic model for gas-particle flows: Analysis of fluidized and moving bed regimes, Chem. Eng. Sci., 80 (2012) 279-292.

[24] S. Cloete, S. Amini, S.T. Johansen, On the effect of cluster resolution in riser flows on momentum and reaction kinetic interaction, Powder Technology, 210 (2011) 6-17.

[25] L. Huilin, D. Gidaspow, Hydrodynamics of binary fluidization in a riser: CFD simulation using two granular temperatures, Chem. Eng. Sci., 58 (2003) 3777-3792.

[26] C.Y. Wen, Y.H. Yu, Mechanics of Fluidization, Chem. Eng. Prog. S. Ser., 62 (1966) $100-111$.

[27] $\mathrm{H}$. Iddir, H. Arastoopour, Modeling of multitype particle flow using the kinetic theory approach, AIChE J., 51 (2005) 1620-1632.

[28] S. Patankar, Numerical Heat Transfer and Fluid Flow, Hemisphere Publishing Corporation1980.

[29] B.P. Leonard, S. Mokhtari, ULTRA-SHARP Nonoscillatory Convection Schemes for High-Speed Steady Multidimensional Flow, NASA TM 1-2568 (ICOMP-90-12)NASA Lewis Research Center, 1990.

[30] A. Yan, J. Zhu, Scale-Up Effect of Riser Reactors (1) Axial and Radial Solids Concentration Distribution and Flow Development, Ind. Eng. Chem. Res., 43 (2004) 58105819.

[31] A. Yan, J. Zhu, Scale-Up of Riser Reactors: Particle Velocity and Flow Development, AIChE J., 51 (2005) 2956-2964.

[32] T. Li, S. Benyahia, J.-F. Dietiker, J. Musser, X. Sun, A 2.5D computational method to simulate cylindrical fluidized beds, Chem. Eng. Sci., 123 (2015) 236-246.

[33] J.X. Zhu, G.Z. Li, S.Z. Qin, F.Y. Li, H. Zhang, Y.L. Yang, Direct measurements of particle velocities in gas-solids suspension flow using a novel five-fiber optical probe, Powder Technol., 115 (2001) 184-192. 
[34] S.F. Foerster, M.Y. Louge, H. Chang, K. Allia, Measurements of the collision properties of small spheres, Physics of Fluids, 6 (1994) 1108-1115.

[35] A. Lorenz, C. Tuozzolo, M.Y. Louge, Measurements of impact properties of small, nearly spherical particles, Experimental Mechanics, 37 (1997) 292-298.

[36] M. Sommerfeld, N. Huber, Experimental analysis and modelling of particle-wall collisions, Int. J. Multiphase Flow, 25 (1999) 1457-1489.

[37] S. Cloete, A. Zaabout, S.T. Johansen, M. van Sint Annaland, F. Gallucci, S. Amini, The generality of the standard 2D TFM approach in predicting bubbling fluidized bed hydrodynamics, Powder Technology, 235 (2013) 735-746.

[38] S. Cloete, A. Zaabout, S.T. Johansen, M. Van Sint Annaland, F. Gallucci, S. Amini, The effect of frictional pressure, geometry and wall friction on the modelling of a pseudo-2D bubbling fluidized bed reactor, Powder Technol., With co-authors (2013). 